\title{
Recent Strategies in Resveratrol Delivery Systems
}

\author{
Noelia D. Machado, ${ }^{[a, b]}$ Mariana A. Fernández ${ }^{[a, b]}$ and David Díaz Díaz ${ }^{*[, d]}$ \\ [a] Dr. N. Machado, Prof. Dr. M. A. Fernández \\ Universidad Nacional de Córdoba, Facultad de Ciencias Químicas, Departamento de Química Orgánica, Ciudad Universitaria, \\ X5000HUA, Cordoba, Argentina. \\ [b] Dr. N. Machado, Prof. Dr. M. A. Fernández \\ Instituto de Investigaciones en Físico-Química de Córdoba (INFIQC-CONICET), Ciudad Universitaria, X5000HUA, Cordoba,Argentina \\ [c] Prof. PD Dr. D. Díaz Díaz \\ Institute of Organic Chemistry, University of Regensburg, Universitätstrasse. 31, D-93040 Regensburg (Germany) \\ E-mail: David.Diaz@chemie.uni-regensburg.de \\ [d] Dr. D. Díaz Díaz \\ Instituto de Productos Naturales y Agrobiología del CSIC, Avda. Astrofísico Francisco Sánchez 3, 38206 La Laguna, Tenerife (Spain) \\ E-mail:d.diaz.diaz@ipna.csic.es
}

Abstract: Resveratrol, a natural polyphenolic stilbenoid widely found in grapes and wines, displays beneficial properties such as cardio protective, antioxidant and anti-inflammatory activities. The trans-resveratrol (RSV) is the most bioactive and more abundant stereoisomer found in nature. Despite the positive properties of RSV, there are various factors that limit its effectiveness, including low aqueous solubility, low oral bioavailability and chemical instability. During the last years, an increasing number of strategies such as nano and micro encapsulation have been developed in order to overcome these limitations and enhance the use of RSV in nutritional and pharmaceutical applications. This review summarizes the advances and main properties of several RSV carriers and delivery systems reported during the last 5 years.

\section{Introduction}

Resveratrol (3,5,4'-trihydroxy-stilbene) is a natural polyphenolic compound belonging to stilbene family that it is normally found in seeds, wines and skin of grapes, soybeans, cherrys, nuts and pomegranates. ${ }^{1}$ The transresveratrol (RSV) is the most active and more abundant stereoisomer found in nature (Figure 1).

The interest of scientists in the utilization of this nutraceutical ${ }^{2}{ }_{3}$ compound has gradually increased during the last years due to apparent beneficial effects in human health, including anticancer, cardio protective, antioxidant and anti-inflammatory activities, among others. ${ }^{3}$.lthough it is present

in several foods, its systemic absorption in the organism is rather limited; being absorbed less than $1 \%$ of consumed RSV. ${ }^{4}$ The main factors that limit the RSV effectiveness are its low aqueous solubility $(<0.05 \mathrm{mg} / \mathrm{mL})$ [1], low oral availability, and chemical instability. The diminished oral bioavailability is due to the susceptibility to suffer sulfatation and glucuronidation during the phase II of metabolism in the gastrointestinal tract. ${ }^{5}$

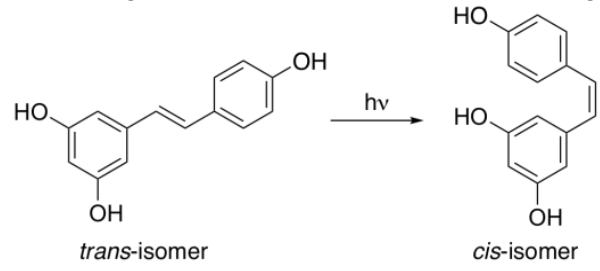

Figure. 1 Trans-to-cis photoisomerization of RSV under UV irradiation.

Furthermore, the trans-to-cis photoisomerization of RSV constitutes another concern, since ca. 80-90\% of the trans-RSV is isomerized when it is exposed to sunlight, white light of high intensity, or ultraviolet light (Figure 1). ${ }^{6,7}$ In addition, high temperatures, ${ }^{8} \mathrm{pH}$ changes ${ }^{9,10}$ and some enzymes, ${ }^{11,12}$ also promote chemical degradation of RSV.

The mentioned factors limit a greater use of RSV in food and pharmaceutical industries, ${ }^{13}$ motivating worldwide research to overcome the aforementioned disadvantages. Thus, during the last decade, numerous research groups have developed and explore potential solutions based on nano and microencapsulation technologies. Within this context, several carriers based mainly on emulsions, particles, gels, nanocrystals and vesicles have been developed. The aim of this review is to provide an overview of the last improvements obtained in the formulation of RSV for different applications. The review is focused on the results published during the last five years, complementing previous revisions ${ }^{3,13,14,15}$. In addition, we also provide a supplementary table (see ESI) that compiles all the chemical structures of the compounds mentioned in the text, which offers a quick visual insight about the molecular structures of the components used to prepare the delivery vehicles.

\section{Delivery systems}




\subsection{Vesicles}

\subsubsection{Niosomes}

Among different carriers, liposomes and niosomes are well-documented drug delivery systems. Niosomes are vesicles consisting of an aqueous core enclosed within a non-ionic surfactant bilayer. Structurally, they are similar to liposomes and posses similar physical properties, so they have been proposed as a stable and less expensive alternative version of those. Following, we will describe some recent applications of niosomes as RSV carriers.

In 2015, Pando and co-workers designed niosomal formulations loaded with RSV for topical use. ${ }^{16}$, The niosomes were prepared using Gelot $64(\mathrm{G} 64)$ as surfactant and oleic (OA) or linoleic acids as stabilizers. In addition, these fatty acids are known as skin penetration enhancers. Different surfactant:stabilizer weight ratios were studied in the range of 1:0.5-1:1.5, with the RSV concentration adjusted to $1 \mathrm{~g} / \mathrm{L}$. The niosomes were prepared using the thin film hydration (TFH) combined with sonication and ethanol injection method (EIM). In both methods, niosomes were spherical in shape, albeit those obtained via EIM were in the range $299-402 \mathrm{~nm}$ and showed polidispersity indexes (PDI < 0.3). On the other hand, the TFH-sonication method produced larger niosomes (ca. 293-496 nm) and with higher PDI indexes $(<0.4)$. Moreover, the RSV encapsulation efficiency (EE) obtained using EIM was higher than THF-sonication (e.g., for formulation G64-OA (1:1), EE = $35 \pm 2 \%$ and $24 \pm 2 \%$, respectively. Finally, stability studies over time using G64/fatty acid (1:1) showed that EIM niosomes were more stable than TFH-sonication ones. The latter showed creaming phenomena with an increase of backscattering up to $20 \%$.

In another example from the same group, Pando and co-workers formulated RSV entrapped niosomes for oral administration. Niosomes were composed by Span 60 or Maisine 35-1 (Mai) as surfactants and dodecanol (Dod) as stabilizer. It was previously demonstrated, that fatty alcohols could also stabilize niosome membranes. ${ }^{17}$ In this study, the RSV concentration in all formulations was adjusted to $0.15 \mathrm{~g} / \mathrm{L}$. Niosomes were prepared using a TFH-sonication method. Among different agitation speeds and surfactant:Dod weight ratio, the optimal values to achieve the highest RSV EE were $15000 \mathrm{rpm}$ and a ratio Span 60:Dod/1:1.5 (w/w), respectively. In addition, the use of these parameters provided niosomes with the smallest mean diameter of 168 $\pm 4 \mathrm{~nm}$, a PDI of $0.247 \pm 0.018$ and an EE of $64 \pm 8 \%$. On the other hand, Mai:Dod/1:1.5 (w/w) niosomes were obtained with a mean diameter of $175 \pm 13 \mathrm{~nm}$, a PDI of $0.260 \pm 0.025$ and a RSV EE of $53 \pm 8 \%$. Stability of niosomes was also determined by analyzing the $\xi$-potential over time. The results indicated that the formulation Span 60:Dod/1:1.5 (w/w) were highly stable, with a $\xi$-potential value of $(-50.1 \pm 0.8) \mathrm{mV}$, confirming the absence of coagulation/flocculation processes due to the electrostatic repulsion effects among the niosomes. Interestingly, in spite of an observed creaming phenomenon, Mai:Dod/1:1.5 (w/w) niosomes exhibited acceptable stability with a $\xi$-potential value of $-39.9 \pm 0.3 \mathrm{mV} .{ }^{18}$

Additionally, Vankayala and co-workers prepared biocompatible RSV-loaded niosomes via sonication method using non-ionic surfactants and a fatty alcohol as stabilizer. ${ }^{19}$ The formulation was prepared with Span 60 and cetyl alcohol in 1:0.5 molar ratio. Niosomes were spherical and exhibited a smooth surface, characterized by $108 \pm 3 \mathrm{~nm}$ particle size with a PDI of $0.24 \pm 0.01$, and $-52.8 \pm 0.6 \mathrm{mV}$ of $\xi$-potential. The RSV EE in this system was $81 \pm 2 \%$, being the encapsulation of RSV confirmed by Fourier-transform infrared spectroscopy (FT-IR) and differential scanning calorimetry (DSC) measurements. The amount of RSV encapsulated by this system was promising for further application of this formulation.

\subsubsection{Liposomes}

Liposomes are the pioneer in vesicular systems to carry active principles. Differing from niosomes, liposomes are vesicles formed by phospholipids, which can include several other constituents like cholesterol to improve the stability of the bilayer.

Caddeo and co-workers developed liposomes for topical delivery of RSV. Liposomes were made from Soy phosphatidylcholine and OA mixed with penetration enhancers (10:1:1 weight ratio), Oramix ${ }^{\mathrm{TM}}$ CG110 (OrCG) and Lauroglycol ${ }^{\mathrm{TM}}$ FCC (LauFCC). ${ }^{20}$. These are dermo-compatible and solubilizing non-ionic surfactants. Liposomes were formed by hydration of the mixture with Tris Buffer at $\mathrm{pH} 7.4$ and sonication at high intensity. Microscopy images showed small and spherical unilamellar vesicles coexisting with oligolamellar structures (Figure 2). RSV vesicles were ca. $90 \mathrm{~nm}$ in diameter and displayed a narrow size distribution (PDI $\leq 0.26 \pm 0.02$ ). The $\zeta$-potential was large and negative, in the range of $-28 /-35 \mathrm{mV}$, due to the presence of $O A$, which prevented vesicle aggregation by superficial electrostatic repulsion. All vesicles presented good RSV EE $(>70 \%)$. In addition, the results of aggregation efficiency indicated that approximately $70 \%$ of phospholipids were assembled into vesicles. MTS cell proliferation assay using 3T3 fibroblasts cells showed quantitative cell viability after $24 \mathrm{~h}$, indicating non-cytotoxicity of liposomes formulations. The presence of RSV inside the vesicles was verified by ${ }^{1} \mathrm{H}$ NMR using the RSV aromatic protons as reference. In contrast to RSV solution or RSV dispersed with empty vesicles (physical mixture), when RSV-loaded vesicles were used, the signals of aromatic protons 
became broader with lower intensity, which was attributed to the slower molecular motion of RSV inserted in the bilayer.

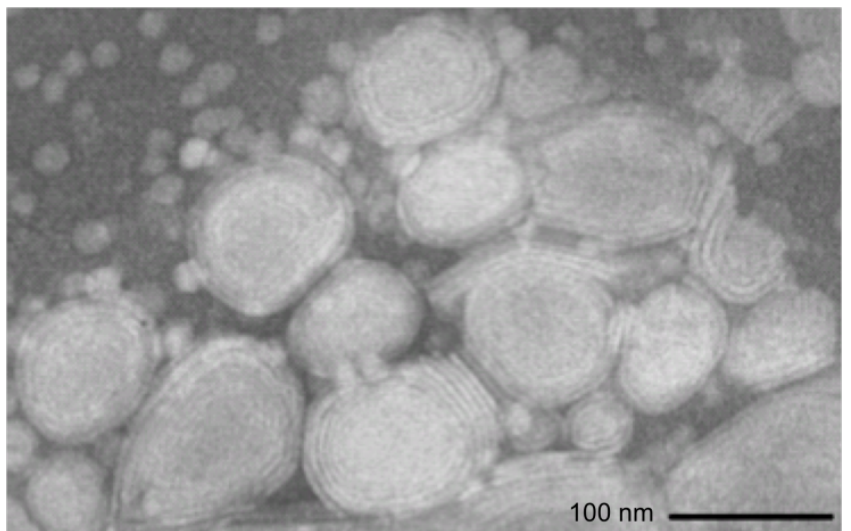

Figure 2. TEM micrographs of RSV-loaded liposomes containing LauFCC (a penetration enhancer). Adapted with permission from ref. 20. Copyright 2015, Elsevier.

Another liposomal formulation coated with succinyl chitosan for oral delivery of RSV was also developed by Caddeo and co-workers..$^{21}$ Liposomes were made of soybean lecithin and OA (10:1 w/w), and $2 \%$ w/w of RSV was added to form the corresponding loaded liposomes. Quercetin was coencapsulated with RSV (Figure 3). In this approach, sonication was employed to prepare the vesicles, and $\mathrm{N}$-succinyl chitosan was used as protective shell of liposomes due to its $\mathrm{pH}$ response $(0.5 \% \mathrm{w} / \mathrm{v})$. Regarding the physical chemical characterization, the coated liposomes were fairly spherical with a mean diameter of $82 \pm 3 \mathrm{~nm}$, a PDI of 0.10 and negative $\zeta$-potential of $-41 \pm 7 \mathrm{mV}$. In this system, RSV EE was $70 \pm 5 \%$. Additionally, small angle $X$ ray scattering experiments confirmed that neither the vesicle lamellar arrangement nor the thickness were altered significantly as a result of the modification with succinyl chitosan, thus confirming the formation of a protective shell. Furthermore, liposomes were stable when stored over two months at different temperatures showing no important variations in their physical chemical characteristics. Very interestingly, accelerated stability profiles showed that the presence of succinyl chitosan increased the physical stability of the formulation in comparison with conventional liposomes, which showed vesicle aggregation.

The same authors reported later the preparation of polyethylene glycol (PEG)-modified liposomes for RSV delivery. ${ }^{22}$ The liposomes were prepared by direct sonication of Phospholipon 90G aqueous dispersion in the presence of two PEG-surfactants, a tri-block polymer L64 (Pluronic ${ }^{\circledR}$ L64) and D- $\alpha$-tocopheryl polyethylene glycol 1000 succinate (TPGS). So-prepared PEG-modified liposomes were spherical with predominant unilamellar morphology, a size of $86 \pm 3 \mathrm{~nm}$, good homogeneity (PDI $0.20 \pm 0.03$ ), and negative $\xi$-potential $(-21$ $\pm 4 \mathrm{mV}$ ). In this case, the EE was ca. $95 \%$. Besides, a greater stability of the vesicles was observed after 2 months of storage at $25{ }^{\circ} \mathrm{C}$ with no signs of physical alteration. In contrast, conventional liposomes showed progressive aggregation and precipitation suggesting that PEG-surfactants improved the vesicles stability.

In general, the above-described modified liposomal formulations showed great features in terms of EE and improved stability compared to conventional ones.

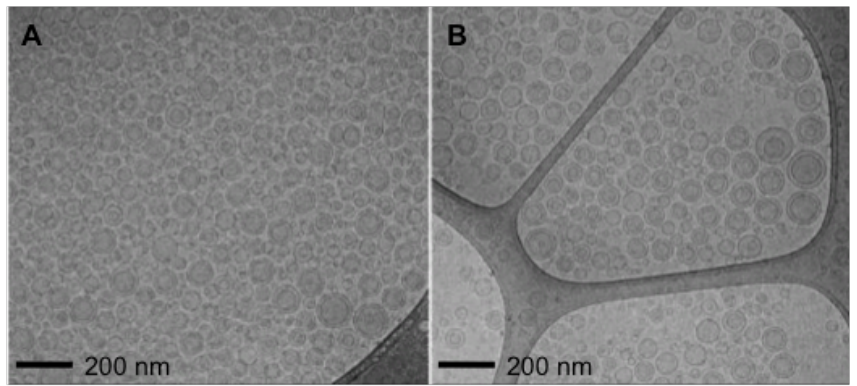

Figure 3. Cryo-TEM images of A) quercetin and resveratrol co-loaded liposomes and B) succinyl-chitosan liposomes. Adapted with permission from ref. 21. Copyright 2017, Elsevier.

\subsection{Emulsions}

\subsubsection{Simple emulsions}


In general, emulsions are colloidal dispersions, typically formed from emulsifiers dispersed in oil, and water. These systems can be distinguished according to their particle radius. In the nanoemulsions, the particle size is smaller than $100 \mathrm{~nm}$ and particles higher than $100 \mathrm{~nm}$ are present in conventional emulsions. Oil-in-water (O/W) emulsions are formed by spherical lipid particles dispersed in an aqueous medium. These particles have a coreshell structure where the nucleus is formed by oil molecules and the hydrophobic chains of the surfactants (i.e. emulsifying agents). The polar layer, instead, is formed by the polar heads of the surfactants. ${ }^{23_{1}}$

In this field of knowledge, Zhou and co-workers developed series of nanoemulsions with the aim to enhance the oral bioavailability of RSV using intestinal glucoronidation inhibitory excipients, i.e. Labrasol..$^{24}$ The oily phase was composed by soybean oil and the aqueous phase was formed by soy lecithin, Labrasol and water. In this case, $0.1 \%$ of RSV was added in the oily phase affording spherical droplets of $132 \pm 9 \mathrm{~nm}$ in diameter, a PDI of $0.18 \pm 0.01$, a $\zeta$-potential of $-49 \pm 4 \mathrm{mV}$. Herein, RSV EE was estimated in $93 \pm 2 \%$, and Labrasol-nanoemulsions showed good stability up to 1 month storage at ca. $4{ }^{\circ} \mathrm{C}$ and $25^{\circ} \mathrm{C}$.

Mucoadhesive nanoemulsions were developed by Nasr for co-encapsulating RSV and curcumin (CUR) for transnasal treatment of neurodegenerative disease..$^{25}$, The oily phase of these nanoemulsions was formed by Labrafac Lipophile and the aqueous phase by $10 \%$ of Cremophor ${ }^{\circledR} 40$ as surfactant. Hyaluronic acid (HA) was incorporated in the aqueous phase at a concentration of $1.5 \% \mathrm{v} / \mathrm{v}$. This compound was selected as coated material to increase the stability and mucoadhesiveness of the formulation. RSV loaded nanoemulsions were formed using a concentration of $0.125 \% \mathrm{w} / \mathrm{v}$. The HA coated formulations were spherical with a particle size of $115.2 \pm 0.2 \mathrm{~nm}$ (Figure 4), a $\xi$-potential value of $-24 \pm 2 \mathrm{mV}$ and a PDI of $0.24 \pm 0.01$. The HA coating significantly increased the particle size and the negative charge of the formulation. In addition, the $\mathrm{pH}$ of nanoemulsions ranged from 6.6-6.7 and HA coated nanoemulsion was able to enhance the mucoadhesive forces ca. 6 times in comparison with uncoated ones. Importantly, the nanoemulsions were found to maintain their properties and resist aggregation after storage at $4{ }^{\circ} \mathrm{C}$. Besides, the amount of RSV did not change significantly and the formulation preserved its anti-oxidant activity even after three months.



Figure 4. TEM micrographs of nanoemulsions composed by Labrafac Lipophile and Cremophor RH 40 (10 \% v/v). A) Uncoated nanoemulsions (magnification: 30000x), and B) $1.5 \%$ v/v HA coated nanoemulsions (magnification: 50000x). Adapted with permission from ref. 25. Copyright 2016, Informa Pharmaceutical Science.

In contrast, Fang and co-workers prepared a RSV delivery system based on emulsions to improve stability and water solubility of RSV. ${ }^{2{ }_{1}}$ The emulsion is formed by 2 -hydroxypropyl- $\beta$-cyclodextrin (2-HP- $\beta$-CD), phosphatidyl choline 60 and a solution of soybean phospholipiddipropylene glycol $(15 \% \mathrm{w} / \mathrm{w})$. The RSVemulsion formulation was prepared by high-pressure homogenization method using $3 \%(\mathrm{w} / \mathrm{w})$ of trans-RSV in a glycerin:water (8:2) mixture. Characterization analyses showed spherical particles with a mean diameter of $122 \pm$ $1 \mathrm{~nm}$ and a PDI of 0.2 . The $\xi$-potential value was $-42 \pm 1 \mathrm{mV}$ and the formulation remained stable over 4 weeks without changes in size and PDI. The $\mathrm{pH}$ of emulsion formulation was $4.99 \pm 0.03$, which is similar to the $\mathrm{pH}$ of human skin.

In another related work, Herneisey and co-workers developed a theranostic RSV-nanoemulsions containing two complementary imaging agents for anti-inflammatory diseases. ${ }^{27}$. These emulsions were prepared with Pluronic ${ }^{\circledR}$ P105, Cremophor ${ }^{\circledR}$ EL and olive oil, and the RSV was incorporated as a solution in polyethylenglycol. The imaging agents used in this study were a near-infrared fluorescence imaging agent, 1,1dioctadecyl-3,3,3',3'-tetramethylindotricarbocyanine iodide, and a ${ }^{19} \mathrm{~F}$ magnetic resonance imaging agent, perfluoropolyether (PFPE). The PFPE was the core material of nanoemulsions, it was surrounded by an organic phase that was capable of carrying RSV and the iodide agent. The mean diameter of the nanoemulsions was $155 \pm 33 \mathrm{~nm}$, with a $\xi$-potential of $-13 \pm 5 \mathrm{mV}$. Besides, the colloidal system remained stable during 348 days stored at 4,25 and $37{ }^{\circ} \mathrm{C}$. Furthermore, no significant difference in RSV loading was found as consequence of PFPE coating (95\% vs. $93 \%$ without PFPE coating).

A different system was developed by Yen and co-workers, who used a self-nanoemulsifying drug delivery system (SNEDDS) for RSV..$^{28}$ The optimized SNEDDS were composed of Capryol ${ }^{\mathrm{TM}} 90$ as the oil phase, Cremophor ${ }^{\circledR}$ EL and Tween 20 as surfactants $(60: 35: 5 \% \mathrm{w} / \mathrm{w} / \mathrm{w})$. In this case, the average particle size was ca. $41 \pm 4 \mathrm{~nm}$ with a PDI of $0.4 \pm 0.1$ (Figure 5 ). 


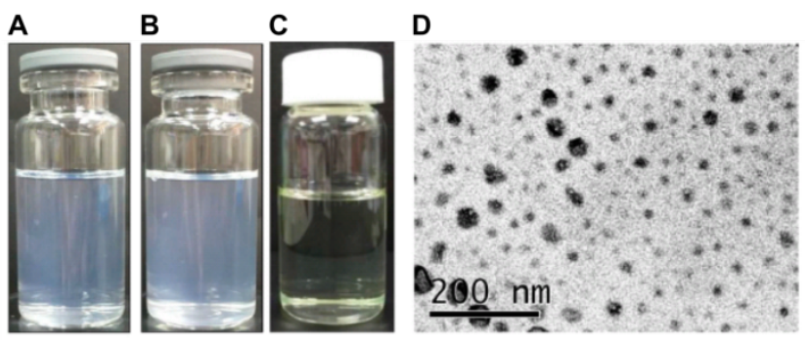

Figure 5. RSV self-nanoemulsifying drug delivery system (RSV-SNEDDS) composed of Capryol ${ }^{\mathrm{Tm}}$ 90, Cremophor® EL, Tween 20 (60:35:5\% w/w/w) dispersed in: A) water; and B) $0.1 \mathrm{~N} \mathrm{HCl}$; C) the appearance of RSV-SNEDDS; and D) TEM micrograph of RSVSNEDDS. Adapted with permission from ref. 28. Copyright 2017, MDPI.

An alternative to this approach was reported by Mamadou and co-workers, who evaluated the capability of SNEDDS to enhance RSV permeation across rat intestine and modulate its presystemic metabolism. ${ }^{29}{ }^{29}$ The semi-solid nanoemulsions (semi-solid NE) were prepared with Gelucire ${ }^{\circledR}$ 44-14 and Labrasol, while liquid nanoemulsions (liquid-NE) were made using Miglyol ${ }^{\circledR} 812$ and Tween 80 . In both cases, a solution of $0.9 \mathrm{mM}$ of RSV was used to prepare loaded nanoemulsions. The semi-solid NE globule size was $21 \pm 5 \mathrm{~nm}$ with a PDI of $0.36 \pm 0.01$ and a $\zeta$-potential value of $-15 \pm 2 \mathrm{mV}$. The liquid-NE had a droplet size of $103 \pm 14 \mathrm{~nm}$, a PDI of 0.39 \pm 0.05 and a $\xi$-potential value of $-15 \pm 2 \mathrm{mV}$.

In comparison, Yang and co-workers prepared a self-microemulsifying drug delivery system (SMEDDS) with uridine- $5^{\prime}$-diphospho-glucuronosyltransferase (UGT) inhibitory excipients to increase the bioavailability of RSV by inhibiting intestinal metabolism..$^{30}$ SMEDDS delivery system was composed of Labrafil, Labrasol and Cremophor RH 40 as oil phase, surfactant and co-surfactants, respectively. $4 \% \mathrm{w} / \mathrm{w}$ of RSV was added to produce loaded SMEDDS, which showed particles with a mean size of $26 \pm 1 \mathrm{~nm}$, a PDI value of $0.23 \pm 0.01$ and a $\zeta$-potential of $-15 \pm 3 \mathrm{mV}$.

O/W emulsions stabilized by quinoa starch particles containing RSV were prepared by Matos and coworkers. Octenyl succinic anhydride (OSA) was used to increase the hydrophobicity of quinoa starch particles. The authors used Tween 20 to stabilize the emulsions and compare them with the effectiveness of starch as a stabilizer. To increase the solubility of RSV, the oil phase was composed of a mixture of Miglyol® 812 and orange oil. The emulsions obtained showed a mean droplet size of $50 \mu \mathrm{m}$ (Figure 6). However, RSV EE obtained for OSA-modified starch granules emulsions was $98 \pm 2 \%$, while Tween 20 stabilized emulsions was $63 \pm 1 \%$. As described by the authors, this could be explained due to RSV solubilization in the surfactant micelles in addition to oil droplets, similar results were also observed for other authors. ${ }^{31}$
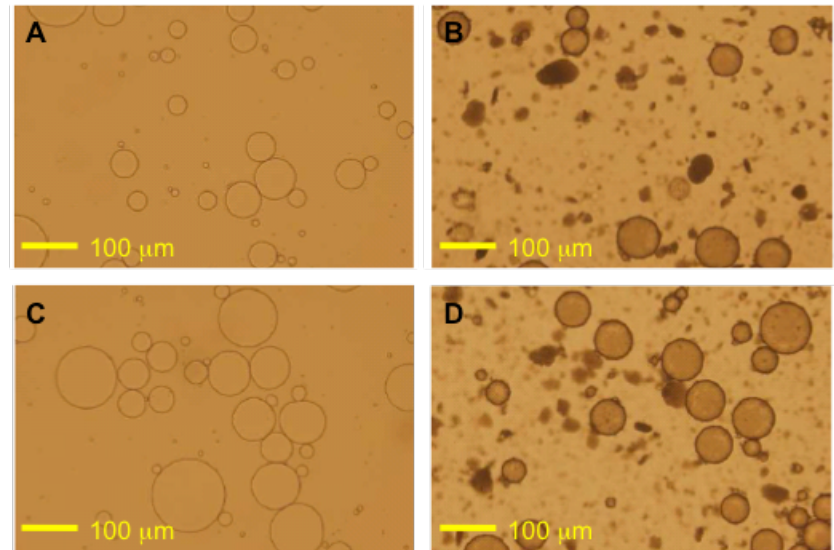

Figure 6. Microscope images of emulsions formulated with pure Miglyol 812 as dispersed phase $(A, B)$ and a mixture of Miglyol 812 and Orange oil (C, D) stabilized with Tween 20 (A, C) and OSA-modified Quinoa starch granules (B, D). Adapted with permission from ref. 32. Copyright 2018, Elsevier.

Regarding the stability of the aforementioned emulsions, Tween 20 stabilized emulsions showed a clear migration of the drops toward the upper part of the cells without increase in droplet size within $1 \mathrm{~h}$. On the other hand, starch emulsions also moved towards the upper part with no changes in drops size. However, the movement was in this case slower than in conventional emulsions, resulting in a more stable one. Authors explained this phenomenon because of the formation of heavier oil drops covered by starch particles than those covered by surfactant. Finally, rotational shear experiments of all samples showed a non-Newtonian behavior due to a marked dependency of the viscosity with shear rate..$^{32,}$

In contrast, Dai and co-workers also designed a SNEDDS for RSV. The formulation was composed of sodium lignin sulfonate/Tween $80 /$ PEG-400 as mixed surfactants and olive oil. ${ }^{33}{ }_{1}$ So-formed spherical particles 
were $119 \pm 5 \mathrm{~nm}$ in diameter with a high $\xi$-potential value of $-22 \pm 1 \mathrm{mV}$. The stability of the formulations was evaluated by heating/cooling cycles. The results showed that lignin containing SNEDDS significantly increased the structural stability of trans-RSV, being the formulation clear, stable, with minor amount of RSV loss $(<2 \%)$, and no phase separation even after $1 \mathrm{~h}$ under direct sunlight.

\subsubsection{Multiple emulsions}

Organized systems such as multiple emulsions of water-in-oil-in-water (W/O/W) are small drops of water trapped within larger oil droplets that are dispersed in an aqueous medium..$^{34}$ In these systems, an oil-soluble emulsifier is usually employed to stabilize the internal water droplets, while a water-soluble emulsifier is used to stabilize the external droplets.

Choi and co-workers formulated a RSV nutraceutical delivery system based on nanoemulsions. ${ }^{35}$. Singlelayer nanoemulsions were prepared by self-assembly emulsification method using water, medium-chain triacylglycerols as oily phase and Tween 80 as surfactant. The double-layer nanoemulsions were prepared adding $0.05 \% \mathrm{w} / \mathrm{v}$ of charged polyelectrolytes (chitosan, alginate or $\beta$-cyclodextrin) onto the system made of single layer nanoemulsions. A RSV ethanolic solution $(5 \mathrm{~g} / \mathrm{L})$ was used to prepare the loaded nanoemulsions. Single-nanoemulsions showed a particle size of $27 \pm 2 \mathrm{~nm}$ and a $\zeta$-potential value of $-9 \pm 1 \mathrm{mV}$. Moreover, double-nanoemulsions had average diameters of $27-55 \mathrm{~nm}$ depending on the polyelectrolyte used. The $\zeta-$ potential of double emulsions prepared with chitosan was $14 \pm 4 \mathrm{mV}$ due to the positive charge of chitosan molecules coated on the surface of nanoemulsions. On the other hand, $\zeta$-potential of double emulsions prepared with alginate was $-23 \pm 1 \mathrm{mV}$ due to the negative charge of the alginate molecules. In addition, $\zeta$-potential of double emulsions with $\beta$-cyclodextrin $(\beta-C D)$ resulted in a stable negative value of $-8 \pm 1 \mathrm{mV}$. To confirm the stability of emulsions, their particle size and $\zeta$-potential were evaluated for 28 days at $25{ }^{\circ} \mathrm{C}$. In general, single and double nanoemulsions prepared with $\beta$-CD were more stable, whereas double nanoemulsions prepared with alginate and chitosan were unstable and showed slight increase in particle size in the same conditions. Furthermore, stability of RSV in nanoemulsions was determined during 7 storage days at $25^{\circ} \mathrm{C}$. The RSV content of double nanoemulsions prepared with $\beta-C D$ was higher than in single-layer nanoemulsions, maintained ca. $95 \%$ of RSV content until 6 days of storage.

Food-grade double emulsion (W/O/W) containing RSV was prepared by Matos and co-workers using conventional and membrane emulsification (ME) methods. ${ }^{36}$, The oily phase of these emulsions was composed by Miglyol ${ }^{\circledR} 812$ containing $5 \%(\mathrm{w} / \mathrm{w})$ of polyglycerol polyricinoleate, a hydrophobic emulsifier. In this work, the inner aqueous phase (W) was composed either by $20 \%$ ethanol $(\mathrm{v} / \mathrm{v})$ or by a RSV solution $(0.05 \mathrm{~g} / \mathrm{L})$ in the same medium. An electrolyte solution formed by $0.1 \mathrm{M} \mathrm{NaCl}$ was added to $\mathrm{W}$ to ensure inner droplets stability. Lastly, the external aqueous phase (W) was composed by $0.5 \%(\mathrm{w} / \mathrm{v})$ of carboxymethylcellulose, $2 \%(\mathrm{w} / \mathrm{v})$ Tween 20 , and $0.1 \mathrm{M} \mathrm{NaCl}$ to balance the osmotic pressure between the two aqueous phases. The best results considering size and distribution, rheological properties, RSV EE and stability, were obtained with ME (Figure 7). In particular, RSV-containing emulsions showed a droplet size of $59.8 \pm 0.2 \mu \mathrm{m}$ and monodispersity (span values in the range 0.87-0.90). Rheological measurements indicated a Newtonian behavior with low elasticity and low viscosity values of ca. $5 \mathrm{mPa}$ s. In addition, RSV EE in these emulsions was ca. $55 \%$. During the formulation storage, the release of RSV from these emulsions was very slow with variations of $2 \%$, suggesting that ME minimizes the drug loss owing to a homogeneous deposition of surfactant at the oil/water interface, which involves a higher barrier against diffusion. Stability measurements showed a slightly instability due to a clarification process as a result of oil droplets rise towards the top of the cell, however this phenomena was reversible.

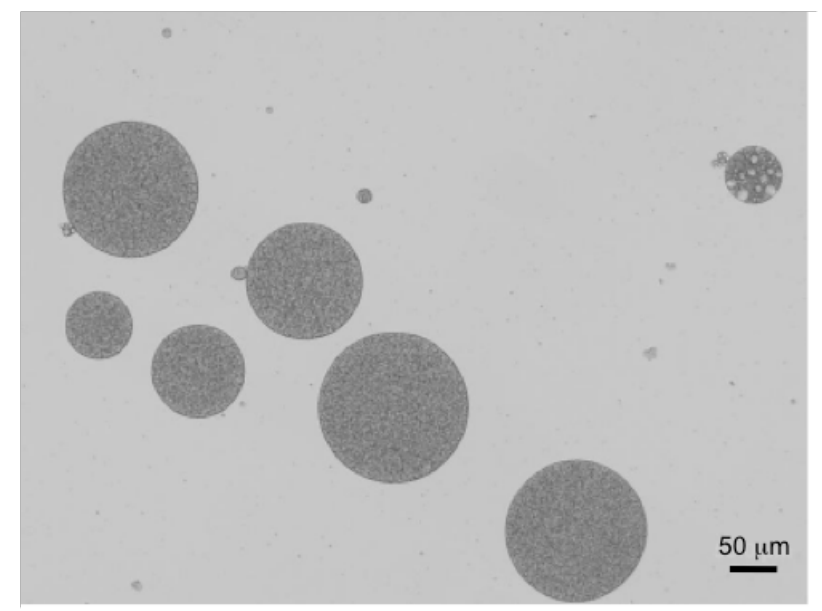


Figure 7. Confocal image of $\mathrm{W}_{1} / \mathrm{O} / \mathrm{W}_{2}$ emulsion containing RSV obtained with UV-vis lamp and prepared by ME. Adapted with permission from ref. 36. Copyright 2015, Elsevier.

In comparison Acevedo-Fani and co-workers formulated another multiple emulsion containing RSV. ${ }^{37}{ }_{j}$ In this case, the single-layer emulsion was composed by lactoferrin and multilayer emulsion was formed by lactoferrin/alginate and lactoferrin/alginate/ $\varepsilon$-poly-L-lysine (Figure 8). Multilayer emulsions were obtained by the electrostatic deposition of the biopolymers using a concentration of RSV of $0.002 \% \mathrm{w} / \mathrm{w}$. Primary emulsions with $1.90 \%(\mathrm{w} / \mathrm{w})$ of lactoferrin exhibited particles with an average diameter of $187 \pm 4 \mathrm{~nm}$, (PDI= $0.15 \pm 0.02)$ and a high positive $\zeta$-potential value of $45.8 \pm 0.5 \mathrm{mV}$. Moreover, secondary emulsions with $0.22 \% \mathrm{w} / \mathrm{w}$ alginate, showed a droplet size of $249 \mathrm{~nm}$ and a very negative $\zeta$-potential value $(-54 \mathrm{mV}) .0 .036 \% \mathrm{w} / \mathrm{w}$ of $\varepsilon$-poly-L-lysine was used to create a third interfacial layer around secondary emulsions. These emulsions showed a droplet size of $416 \mathrm{~nm}$ and a positive $\xi$-potential value of ca. $40 \mathrm{mV}$. Furthermore, the physical stability of emulsions was evaluated by monitoring changes in the droplet size and charge during storage under lightning conditions and at room temperature. Thus, primary emulsions did not show significant changes in average droplet size after 4 weeks. However, secondary emulsions showed a slight size increase from $249 \mathrm{~nm}$ to $274 \mathrm{~nm}$ due to flocculation phenomenon. Finally, tertiary emulsions presented a decrease from $416 \mathrm{~nm}$ to $388 \mathrm{~nm}$, due to rearrangements of $\varepsilon$-poly-L-lysine chains into a denser packing at the interface. Primary emulsions exhibited an important decrease in $\zeta$-potential from $45.8 \mathrm{mV}$ to $15.9 \mathrm{mV}$ after 4 weeks, indicating differences in the net charge of the protein-coated droplets. On the other hand, secondary and tertiary emulsions remained stable during the storage, suggesting an improvement in the stability due to an additional interfacial layer. Morphological studies using transmission electron microscopy confirmed that emulsions showed different microstructures depending on the layer composition. For instance, primary emulsions of lactoferrin-coated oil droplets revealed a well-defined spherical shape surrounded by a lactoferrin layer. The lactoferrin/alginate-coated droplets in secondary emulsions were also spherical with a light inner region and a slightly dark outer region conforming the two-layer coating. The oil droplets coated by three layers instead, had a light core and a visible intense black coating that corresponds to the multilayer coating. Nevertheless, a significant decrease in RSV loading was observed for RSV-loaded oil (control), $53 \%$ after 4 weeks, and it was attributed to the chemical instability of RSV under environmental conditions.
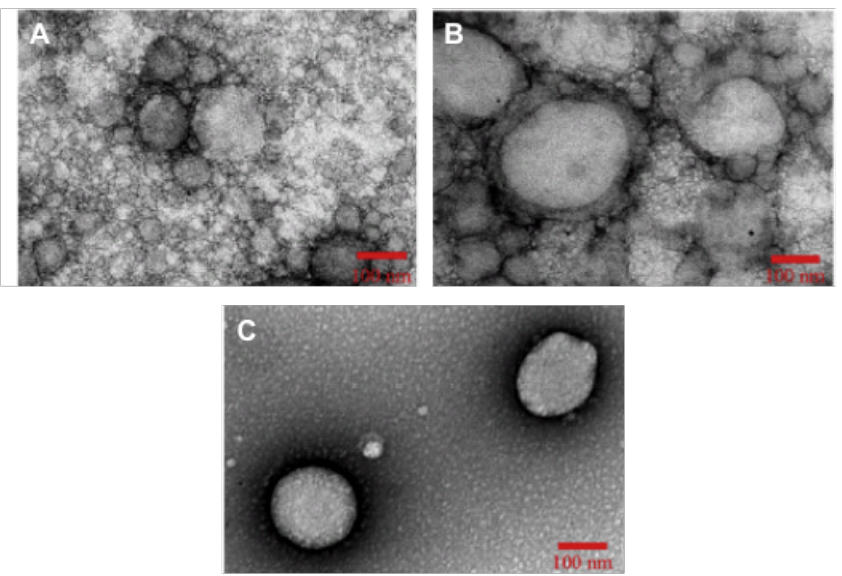

Figure 8. Images obtained by TEM of A) primary emulsions, B) secondary emulsions, and C) tertiary emulsions (magnification: 10000x). Scale bars $=100 \mathrm{~nm}$. Adapted with permission from ref. 37. Copyright 2017, Elsevier.

In a recent work, Matos and co-workers reported the preparation of concentrated W/O/W double emulsions to entrap RSV using a conventional two-step mechanical emulsification process. ${ }^{38}$. The compositions of these double emulsions were the same to the previously reported by the same authors. ${ }^{36}$ Regarding the physicochemical characterization, the formulations presented a bimodal and highly polydisperse droplet size distribution with a mean peak in the 10-30 $\mu \mathrm{m}$ range. However, the samples presented good physical stability since were monitored for 9 days at $30^{\circ} \mathrm{C}$ and no changes were noticed. The EE of RSV was $77.5 \%$. Finally, the double emulsions showed shear-thinning behavior and dominant elastic character.

These results suggest that emulsions are good carriers for RSV delivery due to the good stability of some formulations. In this sense, some single colloidal systems remained stable practically for one year (348 days), whereas in another cases, additional interfacial layers (as in secondary and tertiary emulsions) improved the stability of the systems. The observed variations are mainly related to different composition of the systems.

\subsection{Particles}

\subsubsection{Lipidic particles}


Lipidic particles have a solid hydrophobic core stabilized by a hydrophilic layer of polymers or surfactant molecules. Scalia and co-workers developed lipidic microparticles coated with chitosan medium MW, with the aim to enhance in vivo human skin penetration of RSV. ${ }^{39}$, The microparticles were composed by Tristearin as lipidic material and hydrogenated phosphatidylcholine $(1.4 \%$, w/v) as surfactant. In this example, $6.5-8 \% \mathrm{w} / \mathrm{w}$ of RSV was dispersed in the molten lipid phase. Chitosan is a natural cationic polysaccharide and was selected as coating material due to its biocompatibility and absorption properties, and was used in acetic acid $(1 \% \mathrm{w} / \mathrm{v} ; \mathrm{pH}$ 4.2). Lipidic microparticles (LMs) were prepared using melt emulsification and sonication method. The results showed that obtained LMs were spherical in shape a displayed a smooth surface (Figure 9). On the other hand, mean diameters obtained for uncoated and coated LMs were similar $(6 \mu \mathrm{m})$ while PDI was minor for coated microparticles (PDI: 0.4 vs. 0.14) indicating homogeneous distribution on particles sizes. RSV EE was $67 \%$ for uncoated and $62 \%$ for coated LMs. In addition, $\zeta$-potential values obtained for uncoated LMs was $-18 \pm 5 \mathrm{mV}$, while for coated microparticles was $64 \pm 4 \mathrm{mV}$ due to coated chitosan cationic nature.

Later, Trotta and co-workers developed LMs for RSV delivery to the brain via nose. The LMs were prepared with stearic acid and phosphatidylcholine coated with chitosan $(8.75 \% \mathrm{w} / \mathrm{v}, \mathrm{pH} 4.2)$ using the same methodology aforementioned. These particles were irregular in shape, with a mean diameter of $85 \mu \mathrm{m}$, and presented an encapsulation efficiency of $81 \%$. The $\xi$-potential measurements indicated a high positive value of $45 \pm 3 \mathrm{mV}$ which did not show variation after six months of storage at $4{ }^{\circ} \mathrm{C}$, demonstrating a good stability. ${ }^{[40]}$

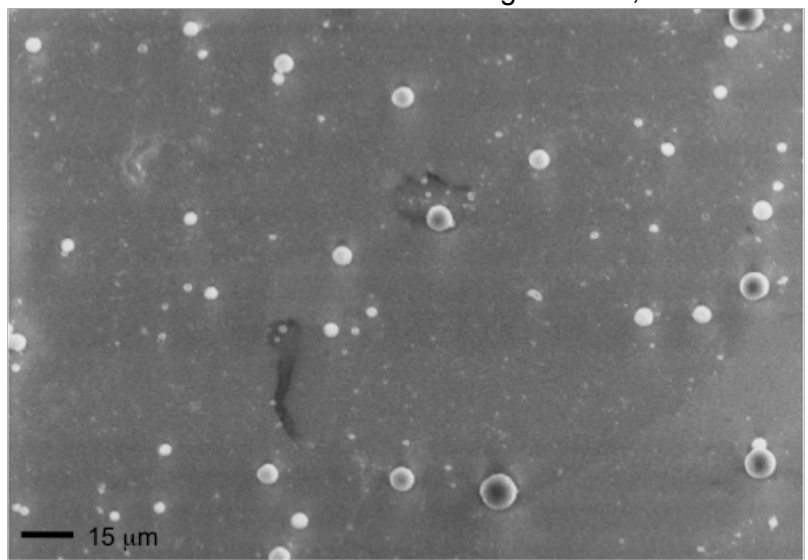

Figure 9. SEM micrograph of chitosan-coated lipidic microparticles loaded with RSV and produced by the optimized procedure. Adapted with permission from ref. 39. Copyright 2015, Elsevier.

Similarly, Wu and co-workers prepared RSV-loaded chitosan nanoparticles via anionic cross-linking for chemotherapy. ${ }^{41}$, The nanoparticles were made of positively charged chitosan and Poloxamer 188. The negatively charged tripolyphosphate was chosen as the cross-linking agent, to improve the stability, solubility and tumors targeting of the natural anti-cancer RSV. RSV-loaded formulations were prepared with $5 \mathrm{~g} / \mathrm{L}$. The obtained particles showed spherical shape with a mean diameter of $217 \pm 10 \mathrm{~nm}$ and a narrow PDI of $0.26 \pm$ 0.03. The $\xi$-potential of RSV-nanoparticles was $59 \pm 6 \mathrm{mV}$, suggesting a high stability of the system. Nevertheless, the EE of RSV was only $11.7 \pm 0.4 \%$.

An evaluation of scavenging capacity and antioxidant damage effect of lipidic nanostructured carriers loaded with RSV was carried out by Jin and co-workers. ${ }^{42}{ }_{1}$ The lipidic nanocarriers for this study were composed by glyceryl behenate, olive oil, Kolliphor EL, TPGS and water. In this case, $0.5 \% \mathrm{w} / \mathrm{w}$ of RSV powder was dispersed in the lipidic mixture. RSV-nanocapsules were prepared by hot melting followed by high pressure homogenization. The particle size was $140 \pm 2 \mathrm{~nm}$, with a PDI of 0.22 and a $\xi$-potential of $-11.2 \pm 0.4 \mathrm{mV}$.

The effect of a chitosan derivative was also evaluated on the surface modification of solid lipidic nanoparticles loaded with RSV, using TMC-g-PA. ${ }^{43}$, TMC- $g$-PA is a grafted cationic polymer formed by $N$ trimethyl chitosan (TMC) modified with palmitic acid. This conjugate forms hydrophobic core-hydrophilic shell micellar structures in aqueous media. The nanoparticles were composed of Precirol ${ }^{\circledR}$ ATO 5, palmitic acid, Gelucire ${ }^{\circledR} 50 / 13$, Tween 80 and $5.45 \% \mathrm{w} / \mathrm{w}$ of RSV and were prepared using hot-melt emulsification and ultrasonication. In addition, the nanoparticles surface modification was performed by simple mixing of the components. Modified RSV-nanoparticles showed larger size than unmodified ones with a mean particle size of $258 \pm 19 \mathrm{~nm}$ vs. $100 \pm 14 \mathrm{~nm}$ and similar PDI values $0.27 \pm 0.05$ vs. $0.32 \pm 0.03$, respectively. Furthermore, positive $\zeta$-potential value was found for modified RSV-nanoparticles $(21 \pm 1 \mathrm{mV})$ in comparison with unmodified nanoparticles $(-19.1 \pm 0.4 \mathrm{mV})$ confirming the surface modification. DSC and FT-IR studies confirmed the encapsulation of RSV, which occurred with an EE of $95 \pm 2 \%$. Stability studies indicated no significant differences in particle size, $\zeta$-potential and EE for modified and unmodified formulations stored at $25{ }^{\circ} \mathrm{C}$ and $4{ }^{\circ} \mathrm{C}$ for three months.

Singh and co-workers also designed solid lipidic nanoparticles loaded with RSV for the treatment of liver cirrhosis. ${ }^{44}$ The nanoparticles were prepared by solvent injection using glyceryl behenate, Gelucire ${ }^{\circledR} 50 / 13$ and 
Tween 80. In this case, $20 \mathrm{~g} / \mathrm{L}$ of RSV was added in the organic phase. The optimized formulations consisted of particles with nearly spherical shape and a mean diameter of $191 \pm 10 \mathrm{~nm}$, PDI $0.16 \pm 0.05$, and $\zeta$-potential of $(-$ $14 \pm 4) \mathrm{mV}$. In this system, RSV EE was established in $74 \pm 4 \%$. DSC and X-ray diffractometry (XRD) studies confirmed the encapsulation of RSV owing to the effect of nanoparticles on the reduction of crystallinity and enhancement of stability.

The preparation of a different kind of nanoparticles functionalized with apolipoprotein $E$ (ApoE) for the delivery of RSV into the brain was reported by Neves and co-workers. ${ }^{45}$, The nanoparticles were prepared using hot-melt emulsification followed by high shear homogenization and sonication. The formulation contained cetylpalmitate, RSV $(5 \mathrm{~g} / \mathrm{L})$ and Tween 80 . The binding of ApoE to nanoparticles surface was carried out by spontaneous interaction between the previously biotinylated ApoE and covalently attached avidin on the particle surface. The obtained ApoE-nanoparticles were almost spherical and uniform in shape with a mean diameter of about $218 \pm 6 \mathrm{~nm}$, with a PDI value of $0.08 \pm 0.030$ and a $\xi$-potential of $-13 \pm 2 \mathrm{mV}$. It is worth mentioning that RSV EE was very high in this system $(98.9 \pm 0.6 \%)$.

Chang and co-workers described another RSV delivery system based on grape peel extract (GPE) and dripping pill (DP) for oral administration. ${ }^{46_{3}}$ The solid dispersion was formed mixing GPE, Capryol ${ }^{\mathrm{TM}}$ 90, Poloxamer 188, Cremophor ${ }^{\circledR}$ EL and PEG 6000. The GPE-DP formulation was prepared using the hot-melt method affording approximately spherical particles with a mean size of $3.53 \pm 0.08 \mathrm{~mm}$, with a RSV content of $1.23 \% \mathrm{w} / \mathrm{w}$. Furthermore, GPE-DP formulation was found to retain $92 \%$ of RSV at $40{ }^{\circ} \mathrm{C}$ and $75 \%$ relative humidity during 6 months.

\subsubsection{Proteic particles}

Koga and co-workers evaluated the stability of RSV encapsulated in microparticles made of proteins using the spray drying method. ${ }^{47}$ A dairy protein such as sodium caseinate and $4.8 \% \mathrm{w} / \mathrm{w}$ of RSV composed the best formulation. The particles, with estimated sizes between 1 and $20 \mu \mathrm{m}$, were no spherical and showed wrinkled surfaces (Figure 10). Besides, RSV microencapsulation efficiency was $68 \%$.



Figure 10. SEM images of RSV microcapsules (microparticles). Adapted with permission from ref. 477. Copyright 2016, Elsevier.

RSV was also encapsulated within soy protein isolate (SPI) nanoparticles using a simple rotary evaporation technique by Pujara and co-workers. ${ }^{48}$ A SPI-RSV complex formed the formulation, for which a RSV solution in ethanol $(1 \mathrm{~g} / \mathrm{L})$ was dropped onto the SPI dispersion. Furthermore, RSV-encapsulated SPI complexes were evaluated for size, $\zeta$-potential, solubility, in vitro drug release and anti-inflammatory activity. The nanocomplexes showed a spherical morphology, with a particle size of $525 \pm 33 \mathrm{~nm}$, a PDI value of $0.56 \pm 0.03$ and a highly negative $\xi$-potential of $(-35.8 \pm 0.6) \mathrm{mV}$. The encapsulation of RSV was confirmed by wide angle XRD and DSC. In both cases, no obvious peaks originating from pure RSV could be observed indicating its amorphous state within the SPI complexes.

Another strategy was reported by Liu and co-workers, who developed a colloidal delivery system based on protein-polyphenol conjugates, protected with a natural surfactant, for RSV and CUR delivery. ${ }^{49}{ }_{1}$ The nanoparticles were fabricated using the antisolvent precipitation method, and a corn oil emulsion in the final step to improve the bioaccesibility. The hydrophobic core was composed by zein-epigallocatechin gallate conjugates, while the shell was made of rhamnolipid (biosurfactant). A corn oil emulsion was used to improve the bioaccesibility. The results revealed that CUR and RSV could be successfully co-encapsulated in the core-shell nanoparticles. The obtained particles were spherical with a mean diameter of $121 \pm 1 \mathrm{~nm}$, a low PDI value of $0.19 \pm 0.01$ and a $\xi$-potential value of $-36.5 \pm 0.1 \mathrm{mV}$ due to the presence of a carboxylic acid group in the molecular structure of the rhamnolipids. Besides, the encapsulation of RSV in an amorphous form was confirmed by spectroscopic analyses. Interestingly, when both polyphenols were simultaneously encapsulated, the EE of RSV was increased from $78 \%$ to $85 \%$. The nanoparticles were relatively stable to aggregation from $\mathrm{pH} 4$ to 9 and at low salt concentration $(<50 \mathrm{mM})$. 
In another work, Guo and co-workers developed hyalurosomes via self assembly of a mixture of oligohyaluronic acid-curcumin polymer and soybean phospholipids, to improve the performance of CUR and RSV. The particles were spherical in shape with a mean diameter of $135 \pm 5 \mathrm{~nm}$ and a negative $\zeta$-potential value of $29 \pm 1 \mathrm{mV}$ at $\mathrm{pH} 7.4$ phosphate buffer solution conditions. The encapsulation efficiency of both polyphenols was $58.8 \%$, and the proposed delivery system was stable during 5 months at room temperature. ${ }^{[50]}$

An interesting proposal was developed by Dai and co-workers, whom prepared lignin nanoparticles loaded with RSV and magnetic nanoparticles $\left(\mathrm{Fe}_{3} \mathrm{O}_{4}\right)$. This green carrier had a diameter of $\sim 160 \mathrm{~nm}$, relatively uniform size distribution and a drug loading efficiency of $90.7 \% .{ }^{[51]}$

\subsubsection{Nanocapsules}

Lipid-core nanocapsules are a specific type of nanocapsules composed of an oily core surrounded by a polymeric shell stabilized by surfactant in aqueous medium. Friedrich and co-workers investigated the behavior of lipid-core nanocapsules loaded with RSV and CUR in human skin penetration..$^{52}$, The oily core of nanocapsules was composed of Span 60 and grape seed oil, whereas the polymeric shell was formed by poly( $\varepsilon$ caprolactone). Tween 80 was used as non-ionic surfactant to stabilize the nanocapsules in aqueous medium, which were prepared by the interfacial polymer deposition method. For the polyphenol-loaded formulations, a concentration of $3.5 \% \mathrm{w} / \mathrm{w}$ was added. RSV-CUR-nanocapsules were characterized by a spherical shape of 201 $\mathrm{nm}$ in diameter, a PDI of 0.09 and a slightly negative $\zeta$-potential value of $-8 \pm 1 \mathrm{mV}$ resulting from the coating by Tween 80. The $\mathrm{pH}$ of suspension was $6.5 \pm 0.1$. The EE was $99.80 \pm 0.02 \%$ and $99.94 \pm 0.04 \%$ for RSV and CUR, respectively. No differences were observed in the physicochemical properties in comparison to single loaded nanocapsules.

\subsubsection{Mesoporous silica nanoparticles}

Summerlin and co-workers encapsulated RSV into mesoporous silica nanospheres (MCM-48) and tested its efficacy in anti-inflammatory and anti-carcinogenic assays. ${ }^{53}$, Nanoparticles were synthesized based on the Stöber method in presence of a surface-active molecule. Tetraethylorthosilicate was used as a silica source and tri-block copolymer F127 (Pluronic F127) acted as particle dispersion agent, whereas cetyltrimethylammonium bromide and ethanol was the structure-directing mixture. $20 \% \mathrm{w} / \mathrm{w}$ of RSV was added to the prepared MCM-48. Physicochemical characterization of nanoparticles showed cubic pores in an ordered array, a size of $283 \mathrm{~nm}$ with a PDI of 0.25 and a $\zeta$-potential of $-22.2 \mathrm{mV}$. RSV encapsulation was verified by XRD due to reduction of nanoparticles surface area after encapsulation and amorphous nature of RVS within nanopores of MCM-48 (RSV free form was crystalline).

Juère and co-workers reported another example of RSV encapsulated in mesoporous silica nanoparticles. The formulation was also made of MCM-48 silica type to encapsulate $20 \% \mathrm{w} / \mathrm{w}$ of RSV. ${ }^{54}$, The nanoparticles had a diameter of $90 \mathrm{~nm}$ with a pore size of $3.5 \mathrm{~nm}$ and were mostly spherical. The encapsulation of RSV was confirmed via wide-angle powder XRD since no reflections associated to the crystallized state of the RSV was detected. In addition, RSV stability from a 10-month-old formulation was studied by UV-Vis spectroscopy and powder XRD. The results confirmed RSV stability against isomerization upon encapsulation.

\subsubsection{Nanocrystals}

Singh and co-workers prepared stable RSV nanocrystals with stabilizers to improve its oral bioavailability. ${ }^{55}$, The nanocrystals were fabricated using probe sonication. The composition of the optimized formulation was TPGS, Lecithin and Pluronic F127 (2:1:0.5) \% w/v. RSV-nanocrystals showed rod like morphologies with a particle size of $(110 \pm 13) \mathrm{nm}$ and a PDI of $0.18 \pm 0.03$ (Figure 11). The $\xi$-potential obtained for this formulation was $-33 \pm 4$ $\mathrm{mV}$. Moreover, the EE of RSV was $94 \pm 2 \%$. In addition, the nanocrystals were stable over a period of 3 months without detriment of their physicochemical properties.

With the aim to improve RSV topical delivery, Sinico and co-workers developed polyphenol nanocrystals with the wet media milling method. In this work, the authors used Poloxamer 188 and Tween 80 as stabilizers. The resultant nanoparticles were spherical and smooth in shape, their size ranged between 0.2-0.3 $\mu \mathrm{m}$ with a homogeneous distribution (PDI, 0.16-0.25). All the formulations presented negative $\zeta$-potential values (-30/-34 $\mathrm{mV})$ and their stability depended on the stabilizer used. Thus, Poloxamer 188-stabilized nanosuspension increased $\sim 100 \%$ their size in 60 days at $25^{\circ} \mathrm{C}$ while Tween 80 ones retained a similar size and PDI values at the same conditions. ${ }^{[56]}$ 


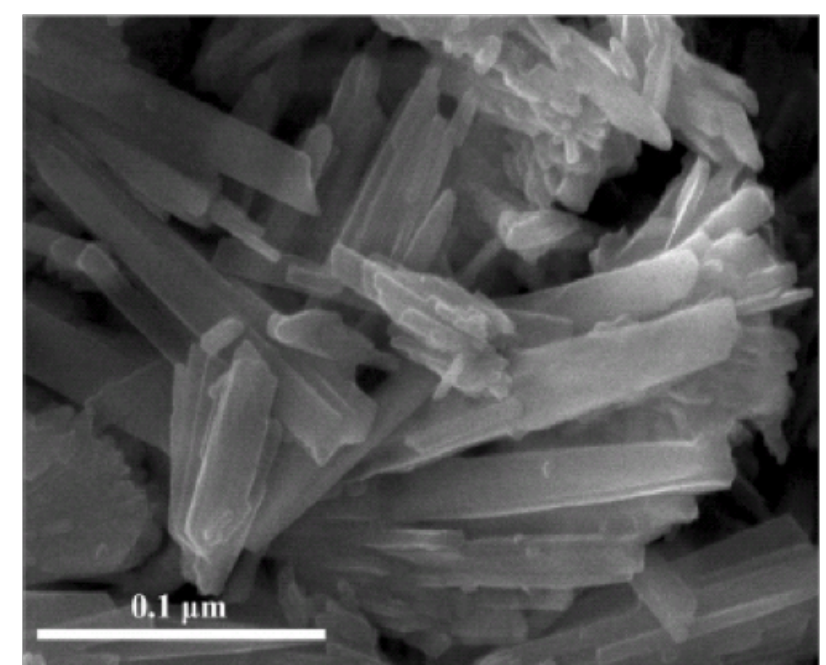

Figure 11. SEM photomicrograph showing the surface morphology of lyophilized trans-RSV nanocrystals. Reproduced with permission from ref. 555. Copyright 2017, Springer.

\subsection{Combining delivery systems}

The combination of several approaches to obtained new carriers constitutes also an interesting alternative to previously mentioned systems are. For instance, Negi and co-workers designed a topical RSV delivery system combining niosomes and hydrogels for treatment of pain disorders. ${ }^{57}$, The hydrogel matrix was prepared with Carbopol ${ }^{\circledR} 934$, due to its hydrophilic and bio-adhesive properties, whereas the niosomes were prepared by TFH using a mixture of Span 80 and cholesterol. The niosomal formulation containing Span 80:cholesterol-2:1 mass ratio and $0.83 \% \mathrm{w} / \mathrm{w}$ of RSV, was found to be the most stable one, showing the lowest sedimentation volume and smallest average particle size $(307.4 \pm 0.3 \mathrm{~nm})$. RSV EE in these niosomes was estimated in $67 \pm 1 \%$. The resulting niosomal hydrogel showed a pseudoplastic behavior (yield value $=63.13 \mathrm{~Pa}$ ) and a pH of 7.1 , which is similar to the $\mathrm{pH}$ of the skin. In addition, the bioadhesive gel was easy to spread and could be extruded from tube.

The co-encapsulation of RSV-CD inclusion complex in the lipophilic and hydrophilic compartments of liposomes was recently developed as a novel RSV carrier (Figure 12). ${ }^{58}$, Liposomes were composed by 1,2dipalmitoyl-sn-glycero-3-phosphocholine (DPPC) and cholesterol at a concentration of $2 \mathrm{mg} / \mathrm{mL}$. In this case, the preparation method was the TFH combined with extrusion. The RSV-CD inclusion complex was formed with 2hydroxypropyl- $\gamma$-cyclodextrin (2-HP- $\gamma-C D)$ at a molar ratio of 2:1. The particle size, PDI and $\xi$-potential of the final formulation were $131 \pm 1 \mathrm{~nm}, 0.089 \pm 0.005$ and $-2.6 \pm 0.5 \mathrm{mV}$, respectively. Moreover, the final formulation was stable at least for 7 days at $4{ }^{\circ} \mathrm{C}$. Importantly, the RSV EE was enhanced ca. 4 times using dual encapsulation strategy compared to conventional liposomes.

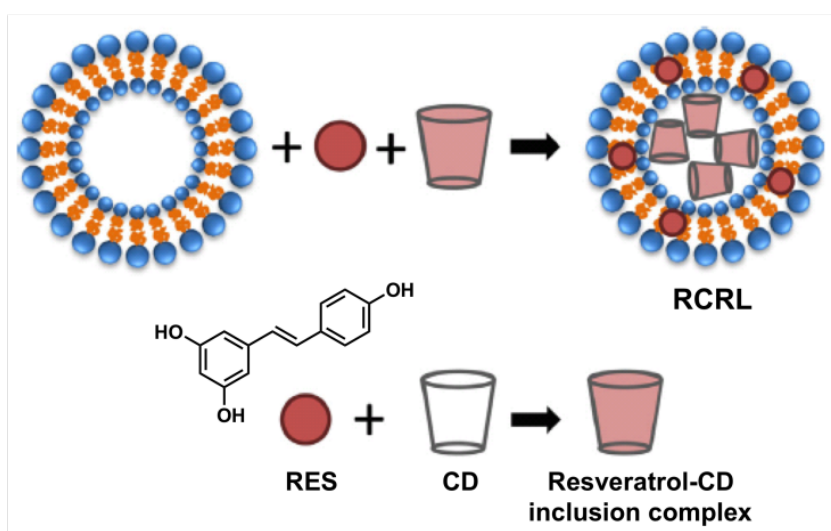

Figure 12. Schematic representation of the RSV delivery system based on a dual nanoencapsulation approach using liposomes and cyclodextrin inclusion complex. Adapted with permission from ref. 58. Copyright 2016, Elsevier.

RSV-CD also was encapsulated in a phospholipid-stabilized nanoemulsions prepared by ultrasoundassisted emulsification. ${ }^{59}$ RSV-CD-nanoemulsions were prepared mixing appropriate amounts of water/oil, lecithin and Tween 80. The resulted formulation showed spherical globules with an average size of $24 \pm 6 \mathrm{~nm}$, a narrow PDI of $0.30 \pm 0.03$ and a high positive $\xi$-potential $(33 \pm 4 \mathrm{mV})$. Moreover, the droplets were stable up to 4 
months with no significant changes observed on particle size and UV-visible absorption of the system. The EE of RSV-CD in nanoemulsions was of $99.95 \%$. Various physicochemical parameters of nanoemulsions were obtained at $37^{\circ} \mathrm{C}$, such as $\mathrm{pH}$, refractive index, surface tension and viscosity. The values obtained were $6.66 \pm$ $0.05,1.356 \pm 0.001 \mathrm{~N}, 27.0 \pm 0.5 \mathrm{mN} / \mathrm{m}$ and $2.3 \pm 0.7 \mathrm{Ns} / \mathrm{m}^{2}$, respectively. These results suggested that the formulation could be used as delivery vehicle since it was slightly acidic, homogeneous and stable, with good spreading and mixing of components and fully dilutable.

Another approach was developed by Hao and co-workers, who reported the in situ fabrication of a gel, using deacetylated gellan gum (DGG), loaded with RSV nanosuspensions for intranasal drug delivery. ${ }^{60}{ }$ DGG is an anionic polysaccharide that can form transparent gels on exposure to certain concentrations of cations found in the nasal mucosa mucus. RSV nanosuspensions were prepared according to the solvent precipitation method. Lyophilized nanosuspensions were introduced in DGG solutions and mixed, to obtain the final formulation. The best formulation contained $0.6 \% \mathrm{w} / \mathrm{v}$ of gellan gum due to favorable gelling ability and desired viscosity. Particle analysis showed spherical shape with a mean diameter of $241 \mathrm{~nm}$ and a PDI of 0.23 . The $\xi$-potential was $-9 \mathrm{mV}$ and the colloidal suspension was stable during 30 days storage at room temperature. Furthermore, the rheological studies indicated that this formulation showed shear thinning and thixotropic properties. In addition, the rheological behavior obtained for the in situ gel revealed adequate mechanical properties for intranasal administration, since high values of adhesiveness were obtained, indicating a prolonged residence time on the application site and low values of cohesiveness, demonstrating good spreading and covering of the formulation over the mucosal tissue.

El-Far and co-workers reported an alternative system. The authors fabricated a multicompartmental nanovehicle based on casein micelles and phytosomes for delivering antineoplasic drugs, monascus yellow pigments and RSV.$^{61}$ A complex between phosphatidylcholine and RSV composed the phytosome. Accurate quantities of pigments and casein were used to prepare the loaded micelles, whose dispersion was then used to hydrate the film of phytosome. Physicochemical characteristics of dual drug-loaded casein micelles revealed smooth spherical morphology, a particle size of $225 \pm 9 \mathrm{~nm}$ with a PDI of $0.21 \pm 0.02$ and a $\xi$-potential value of $54 \pm 5 \mathrm{mV}$. Moreover, RSV was encapsulated with $82 \%$ efficiency. The disappearance of the melting endothermic peak of pure RSV at around $265^{\circ} \mathrm{C}$ in the DSC thermogram confirmed the formation of phytosomal complex. In addition, the particle size was not increased from initial measurements after 3 months of storage at 4 ${ }^{\circ} \mathrm{C}$, confirming the physical stability of the formulation.

\section{Overcoming limitations}

\subsection{Solubility}

There are several systems where the solubility of RSV was increased due its incorporation in different carriers. The most important increment in the solubility of RSV was obtained in the GPE-DP formulation (solid dispersion of the mixture of GPE, Capryol ${ }^{\mathrm{TM}}$ 90, Poloxamer 188, Cremophor ${ }^{\circledR}$ EL and PEG 6000). ${ }^{466}$ In this system the RSV solubility was ca. 14 times higher than in GPE alone (i.e. $63.1 \pm 1.0 \mu \mathrm{g} / \mathrm{mL}$ vs. $903.9 \pm 7.4 \mu \mathrm{g} / \mathrm{mL}$ ). A similar enhancement was observed in RSV nanocrystals stabilized with Tween 80 where the polyphenol solubility increased from $65 \pm 3 \mu \mathrm{g} / \mathrm{mL}$ (for bulk RSV) to $902 \pm 2 \mu \mathrm{g} / \mathrm{mL}$. ${ }^{[56]}$

In contrast, RSV-SPI complex nanoparticles enhanced 2-fold the solubility of RSV in relation with unencapsulated RSV. ${ }^{48}$ Similarly, MCM-48 also enhanced, approximately 2 times, the RSV solubility when it was encapsulated..$^{53}$

\subsubsection{In vitro release studies}

Several in vitro release studies of RSV were carried out using different delivery systems and experimental conditions. In some examples, the systems showed sustained release. Thus, RSV release from succinyl chitosan-stabilized liposomes was $15 \%$ after $2 \mathrm{~h}$ at pH 1.2, and an almost complete release of RSV after $8 \mathrm{~h}$ at $\mathrm{pH} 7.0$ and at $37{ }^{\circ} \mathrm{C}$ was observed. The observed difference could be due to the high solubility of the polysaccharide at neutral $\mathrm{pH} . .^{21}$

In vitro RSV release studies from chitosan nanoparticles were also performed at body temperature $\left(37^{\circ} \mathrm{C}\right)$ and $\mathrm{pH}$ values of 7.4 and 6.5 , to mimic both physiological and tumor tissue conditions, respectively. The release from nanoparticles exhibited a biphasic pattern characterized by a fast initial burst effect during the first $2 \mathrm{~h}$, followed by a slow and sustained release. The accumulated RSV release was $40 \%$ at $\mathrm{pH} 7.4$ and $50 \%$ at $\mathrm{pH}$ 6.5 over a period of $12 \mathrm{~h}$ (Figure 13). These results suggest that the weak acidic condition of tumor tissues is beneficiary for the release of RSV from the nanoparticles. ${ }^{41}$ 


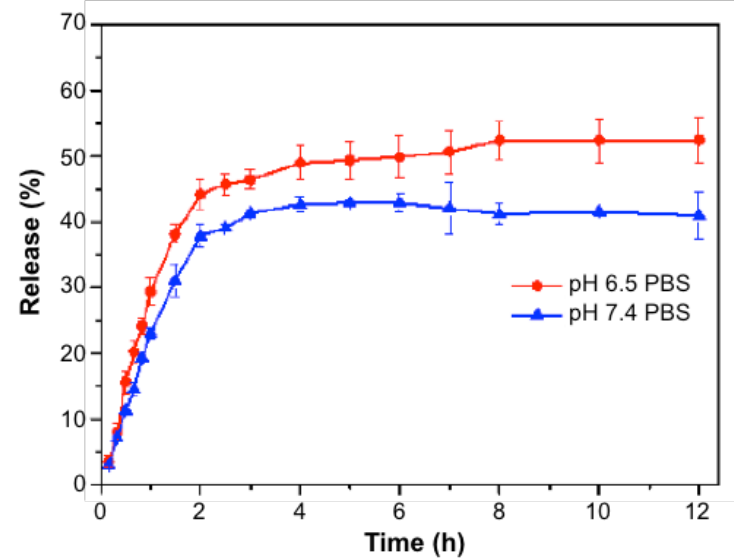

Figure 13. In vitro RSV release profiles of RSV-chitosan particles. Data are means \pm S.D., $n=3$. Accumulated RSV release content in pH 6.5 solution is higher than that of $\mathrm{pH}$ 7.4. Adapted with permission from ref. 41. Copyright 2017, Elsevier.

Similar results were obtained when RSV in vitro release studies were performed at $34{ }^{\circ} \mathrm{C}$ from lipidic nanoemulsions. In these studies ca. $60 \%$ of RSV was released after $6 \mathrm{~h}$ at $\mathrm{pH} 6$ through a diffusion-controlled mechanism (Figure 14)..$^{25}$

Additionally, the in vitro release study of RSV from RSV-CD complex encapsulated in phospholipid nanoemulsions was carried out in phosphate buffer saline at $\mathrm{pH} 7.4$ and $37^{\circ} \mathrm{C} . .^{59}$, The cumulative releases $(\%)$ of RSV and RSV-CD from nanoemulsions were similar (ca. $55 \%$ ) after $15 \mathrm{~h}$, also both release profiles showed fast and then sustained release. The initial fast release could be due to RSV present at the interface of nanoemulsions, which came out into aqueous medium rapidly. In contrast, the slow release could be attributed to the hydrophobic interactions between the oily core of nanoemulsions and RSV, or hydrogen bonding interaction between hydroxyl group of CD and surfactant molecules.

In all these cases, the RSV sustained release was higher and faster from surface modified liposomes. Then, chitosan nanoparticles and RSV-CD complex in nanoemulsions showed a very slow release.

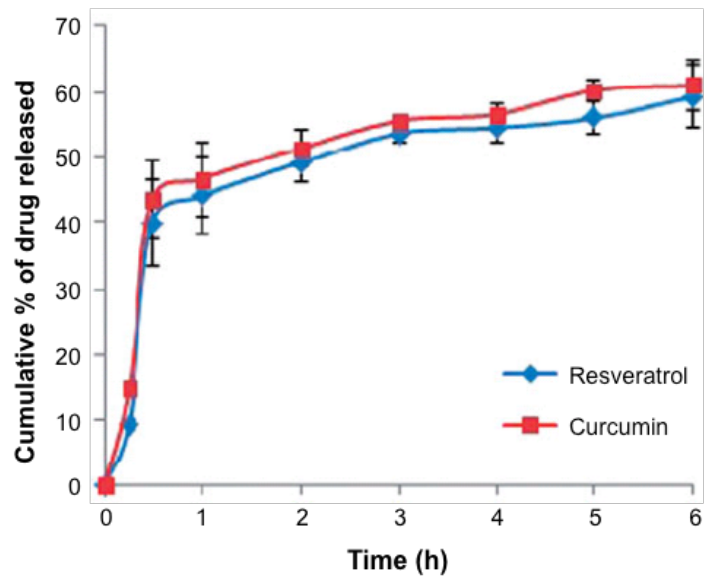

Figure 14. In vitro release of RSV and CUR from the mucoadhesive nanoemulsion coated with HA in phosphate buffered saline pH 6 containing 2 \% Tween 80. Adapted with permission from ref. 25. Copyright 2016, Informa Pharmaceutical Science.

It is well known that delivery systems could reduce the release of diverse encapsulated compounds because of many causes. For example, it could be due to the lipophilicity of the entrapped drug. RSV release from niosomes (Span 60 and cetyl alcohol) in simulated gastrointestinal conditions showed a $30 \%$ and $9 \%$ of $\mathrm{RSV}$ within $2 \mathrm{~h}$ at $\mathrm{pH} 1.2$ from free solution and niosomes, respectively. Later, at $\mathrm{pH} 7.4$, ca. 96\% of RSV was released from its free form within $7 \mathrm{~h}$, while the nanoencapsulated RSV was released ca. $81 \%$ after $12 \mathrm{~h}$ (Figure 15). $\cdot^{19}$ 


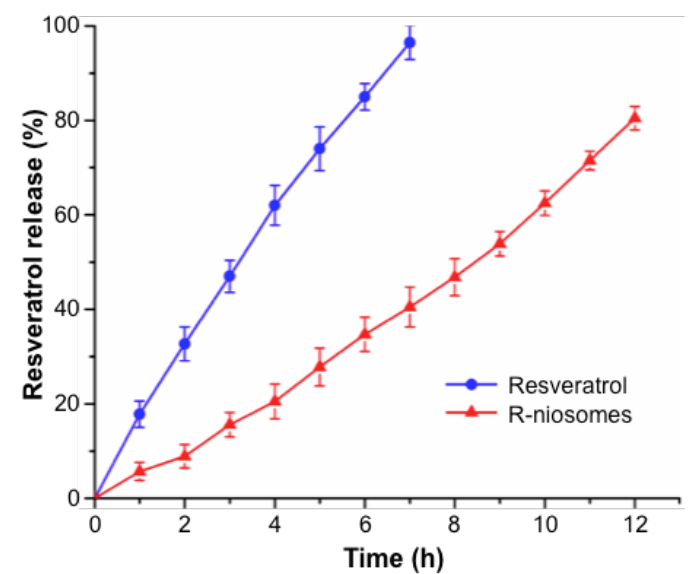

Figure. 15. In vitro release profile of free resveratrol and resveratrol encapsulated in niosomes (R-niosomes). The first $2 \mathrm{~h}$ of dialysis was carried out in a solution maintained at $\mathrm{pH} 1.2$ using $0.1 \mathrm{~N} \mathrm{HCl}$ and it was continued in phosphate buffer solution at pH 7.4 for $10 \mathrm{~h}$, to simulate the gastric and the intestinal conditions, respectively. Adapted with permission from ref. 19. Copyright 2018, Elsevier.

In a similar way, RSV release from nanoemulsions (soybean oil, soy lecithin, Labrasol and water) after $24 \mathrm{~h}$ was significantly slower than from plain solution (60\% vs. $100 \%$, respectively). ${ }^{24}$ Similar results were obtained from nanoemulsions coated with PFPE. The emulsion showed a controlled release of RSV in phosphate buffer saline at $\mathrm{pH} 7.4$ and $37{ }^{\circ} \mathrm{C}$ thus $57 \%$ of RSV was released from this formulation, whereas a maximum of $70 \%$ was obtained for free RSV solution after $285 \mathrm{~h} \cdot .^{27}$ The same tendency was observed from SMEDDS (composed of Labrafil, Labrasol and Cremophor RH 40 as oil phase). The RSV in vitro release was $27 \%$ from SMEDDS, and $80 \%$ was rapidly released from the free RSV suspension within $0.75 \mathrm{~h}$ in water at $37^{\circ} \mathrm{C}$ (Figure 16$)^{30}$,

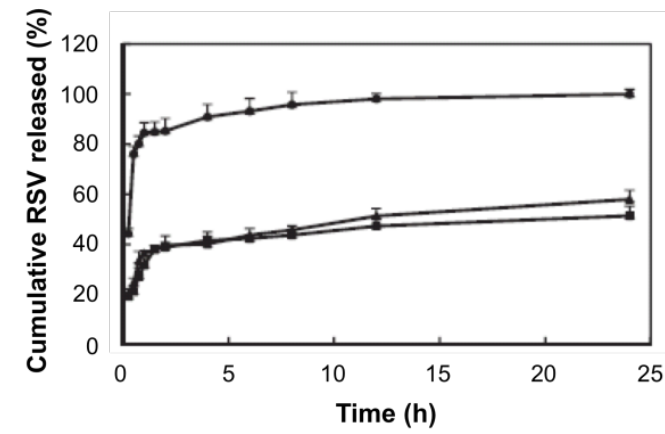

Figure 16 Percentage of RSV released as a function of time from free drug or self-microemulsifying drug delivery system (SMEDDS) $(n=3$, mean \pm S.D). - Free RSV, $\Delta$ SMEDDS containing UGT inhibitory excipients, - control SMEDDS (without UGT inhibitory excipients). Adapted with permission from ref. 30. Copyright 2018, Elsevier.

A nanovehicle based on casein micelles and phytosomes exhibited a biphasic release profile of RSV characterized by initial burst release, followed by a prolonged release phase. After $24 \mathrm{~h}, 72 \%$ of RSV was released from phytosomal-micelles, whereas free RSV was completely released from its solution within $2 \mathrm{~h}$ at $\mathrm{pH}$ 7.4 and $37^{\circ} \mathrm{C} .{ }^{61}$ In the aforementioned examples, the reduction of RSV release was achieved more effectively using nanoemulsions, specifically with PFPE coated ones in comparison with niosomes, microemulsions and phytosomal-micelles.

A reduction of RSV release occasionally is caused by the delivery system surface modification. For example, the in vitro release profile of RSV from PEG-modified liposomes was constant and slower than from conventional liposomes (20\% vs. $45 \%$ after $8 \mathrm{~h}$ ), achieving the same value after $24 \mathrm{~h}(\mathrm{ca} .70 \%) .{ }^{22}{ }$ Hence, the proposed formulation could modulate the release in a time-dependent manner, and PEG-surfactants played an important role in controlling the release rate.

Similarly, lipidic microparticles coated with chitosan showed a ca. $30 \%$ of RSV release in $6 \mathrm{~h}$ whereas $50 \%$ was released from a control formulation (Figure 17). ${ }^{39}$ 


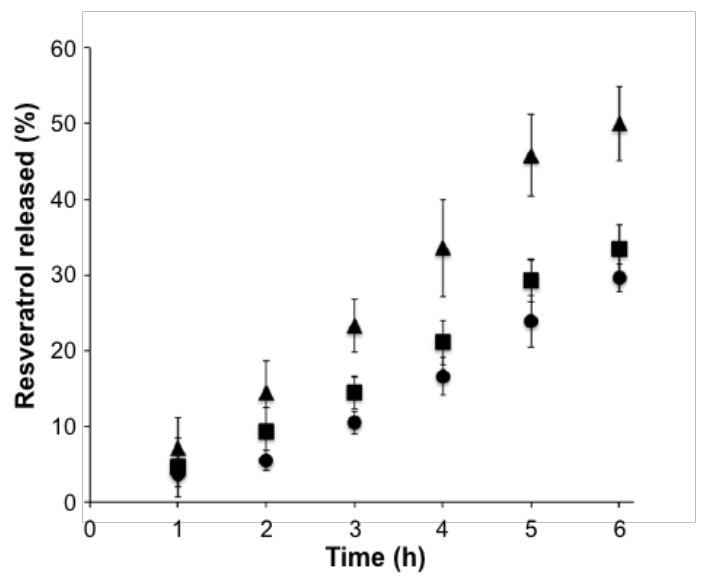

Figure 17. Franz diffusion cell release profiles of free RSV (filled triangles), uncoated RSV-loaded lipidic microparticles (filled squares) or chitosan-coated RSV-loaded lipidic microparticles (filled circles) from their cream formulations. Values are means \pm S.D. ( $n=6$ ). Adapted with permission from ref. 39. Copyright 2015, Elsevier.

In gastrointestinal simulated conditions, RSV solid lipidic nanoparticles, showed an initial burst release at $\mathrm{pH} 1.2$, and sustained release pattern at pH 6.8 after $24 \mathrm{~h}, 74 \pm 4 \%$ and $68 \pm 4 \%$, respectively. When modified lipidic nanoparticles with TMC-g-palmitic acid were used, $43 \pm 2 \%$ of RSV was released in a sustained manner at $\mathrm{pH} 6.8$, whereas it was less than $25 \%$ at $\mathrm{pH} 6.8 .^{43_{1}}$

Solid lipidic nanoparticles ApoE functionalized reduced ca. 1.5 times the RSV release and the initial burst effect was avoided in comparison with non-functionalized ones after $28 \mathrm{~h}$, in blood stream simulated conditions at $37^{\circ} \mathrm{C} .4^{45}$

In the case of niosomal hydrogel system, the slower RSV release in comparison with a suspension could be attributed to higher diffusion limitations in the hydrogel matrix. In this sense, $90 \%$ of RSV was released over 6 $\mathrm{h}$, being only slightly slower from the niosomal hydrogel compared to a suspension formulation. Among different mathematical models, the Korsmeyer-Peppas kinetic model provided the best fitting for both niosomal and suspension formulations. ${ }^{57}$

These results indicate that the surface modification was an appropriate strategy to reduce the RSV release, specifically in liposomes. Indeed, a reduction of 2.2 times was observed, while in the other cases this decreasing was ca. 1.5 times.

In sharp contrast the release of RSV could also be enhanced and in many cases, the enhancement is attributed to the increment in its aqueous solubility. In this sense, in vitro release studies from SNEDDS (made of lignin sulfonate/Tween 80/PEG-400 and olive oil) were performed at $37^{\circ} \mathrm{C}$ and $\mathrm{pH}$ values of 1.2 and 6.8 . Under both simulated conditions, RSV was released faster from lignin SNEDDS (ca. 2 times higher than from pure RSV solution) (Figure 18). Thus, the proposed formulation contributed to improve the dispersion and dissolution of RSV in the gastroenteric environment. ${ }^{33}$ 

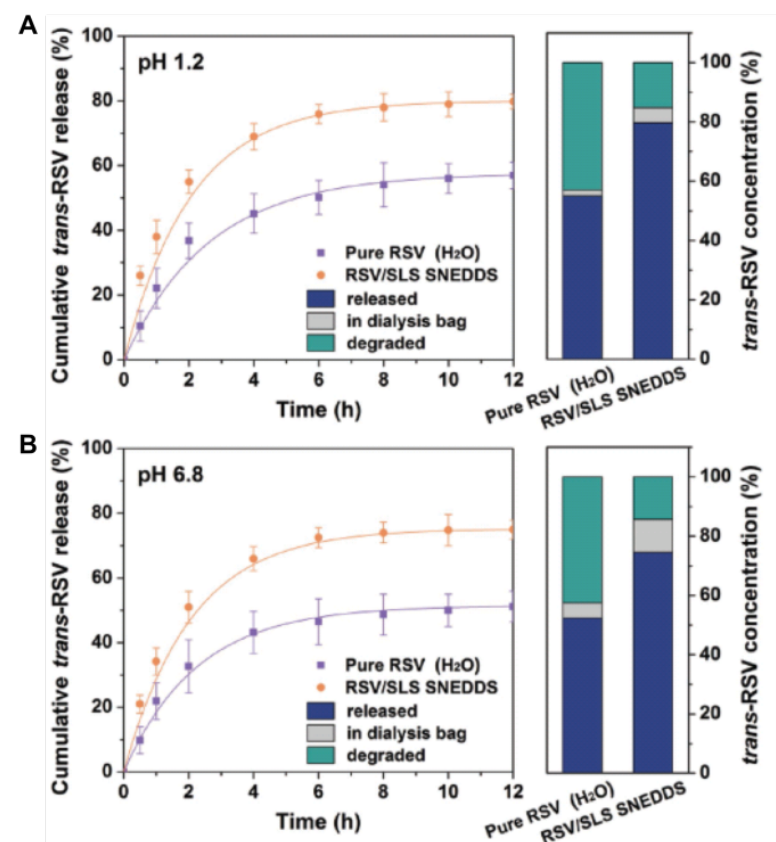

Figure 18. Cumulative trans-RSV release of self-nanoemulsifying drug delivery system (SNEDDS) and pure RSV from dialysis bag in A) $\mathrm{pH} 1.2$ and B) pH 6.8. Adapted with permission from ref. 33. Copyright 2018, John Wiley \& Sons.

Regarding lipidic nanoparticles, some RSV in vitro release studies showed an enhanced release at the same time, in comparison with a control suspension. For example, $80 \pm 4 \%$ of RSV was released from modified nanoparticles while $37 \pm 5 \%$ was released from RSV control suspension at pH 7.4 and $37{ }^{\circ} \mathrm{C}$ after $24 \mathrm{~h}^{4}{ }^{44}$

In comparison, in vitro release experiments were carried out at $37{ }^{\circ} \mathrm{C}$, at $\mathrm{pH} 1.2$ and 6.8 . Final accumulated release of RSV from GPE at pH 1.2 was only $23 \pm 1 \%$, while that from GPE-DP was $67.8 \pm 0.4 \%$. Moreover, at $\mathrm{pH} 6.8$ the values were $14.9 \pm 0.5 \%$ and $64.5 \pm 0.7 \%$ for GPE and GPE-DP, respectively. These results confirmed the significant increase in RSV solubility due to GPE-DP formulation. ${ }^{46_{1}}$

In the same conditions above mentioned, RSV was released from the SPI nanoparticles faster compared to pure RSV solution in both conditions. After $2 \mathrm{~h}$ at $\mathrm{pH} 1.2,60 \%$ of RSV was released from the proposed formulation and only $16 \%$ from pure RSV solution. After $24 \mathrm{~h}$ in intestinal conditions, ca. $50 \%$ of RSV was release from the control solution and ca. $90 \%$ from the nanocomplexes (Figure 19). This faster release can be attributed to its improved solubility because of the encapsulation. ${ }^{48}$

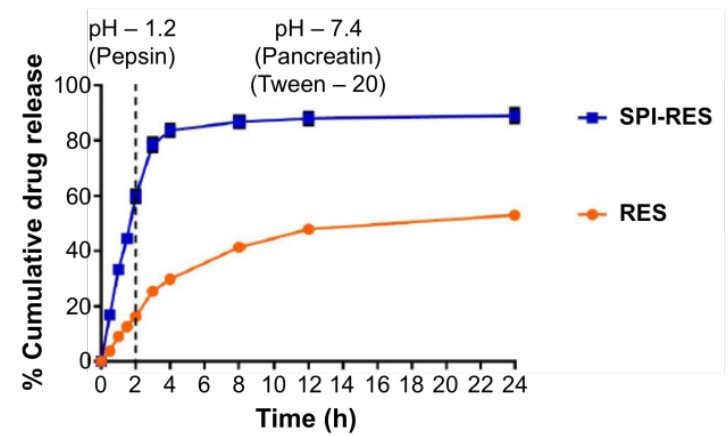

Figure 19. In vitro release of pure RSV and RSV encapsulated in soy protein isolate nanocomplex (SPI-RSV). Mean \pm S.D. ( $n=3)$. Adapted with permission from ref. 48. Copyright 2017, Elsevier.

Another study of in vitro drug release was performed at $\mathrm{pH} 7.4$ and $32{ }^{\circ} \mathrm{C}$ from lipid-core nanocapsules, loaded with RSV, or with RSV-CUR co-encapsulated. After $6 \mathrm{~h}$, no considerable differences were observed in RSV release, i.e. ca. 38 \% from RSV-nanocapsules and ca. $43 \%$ from RSV-CUR-nanocapsules. However, after $24 \mathrm{~h}$, ca. $73 \%$ of RSV was release from RSV-CUR-nanocapsules and ca. 58 \% from RSV-nanocapsules (Figure 20). Such enhancement of the release kinetics suggested a promoted release due to co-encapsulation. ${ }^{52_{1}}$ 


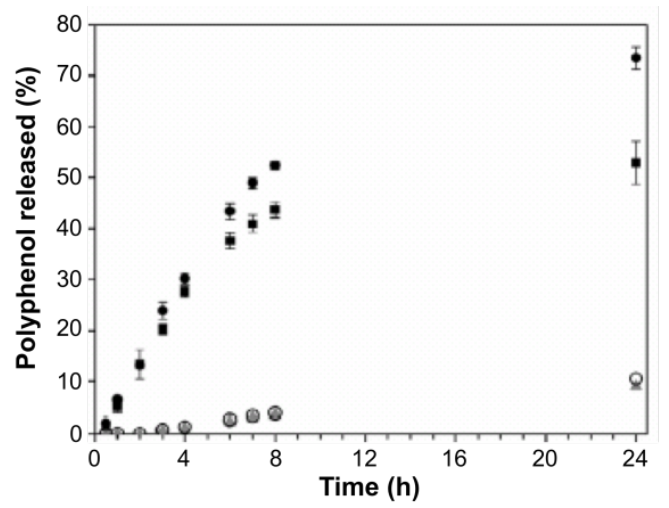

Figure 20. In vitro release profiles of RSV and CUR from lipid-core nanocapsules (LNC) (n=3) at $32{ }^{\circ} \mathrm{C}$. Release from RSV-CUR-LNC is depicted with circles (• RSV; O CUR), whereas - and $\Delta$ depict release curves for polyphenols from RSV-LNC and CUR-LNC, respectively. S.D., which were always below $1.06 \%$, are not shown for CUR curves to maintain a clear data presentation. Adapted with permission from ref. 52. Copyright 2015, Elsevier.

Studies of RSV release from RSV-CD inclusion complex-liposomes were carried out at $\mathrm{pH} 7.4$ and $37{ }^{\circ} \mathrm{C}$ for $24 \mathrm{~h}$. The cumulative release of RSV was almost complete for dual encapsulated liposomes (94\%) compared to its free form (44\%), due to the increased RSV aqueous solubility because of CD inclusion complex..$^{58}$

In vitro dissolution studies carried out at $\mathrm{pH} 7.4$ and $37^{\circ} \mathrm{C}$ with RSV encapsulated in nanoparticles made of MCM-48 silica type showed a $26 \%$ of RSV released from a pure solution, whereas $70 \%$ was released from the proposed formulation (with $\mathrm{MCM}-48$ ) as a consequence of enhanced solubility. The release profile was fitted with the semi-empirical power law model, and the zero-order kinetic model drove the drug dissolution (diffusional exponent of 0.944) [54]. In the same medium ( $\mathrm{pH} 7.4$ and $37{ }^{\circ} \mathrm{C}$ ) in vitro RSV release studies were also performed from nanocrystals. After $5 \mathrm{~h}, 95 \%$ of RSV was released from the formulation whereas only $40 \%$ was released from plain RSV, which could result from the RSV dissolution rate enhancement with nanocrystals..$^{55}$,

In the case of silica nanospheres of MCM-48 studied by Summerlin and co-workers, the faster diffusion and release of RSV was due to the RSV amorphous state and the higher surface area within nanoparticles, since the in vitro release was much slower from RSV in dimethyl sulfoxide solution (31\%) than from MCM-48 (65\%) after $24 \mathrm{~h}$ (Figure 21). ${ }^{53}$

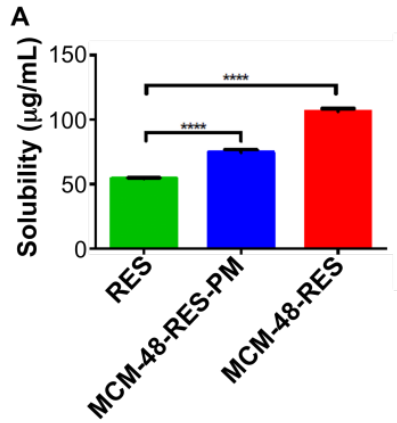

B



Figure 21. A) Saturated aqueous solubility of pure RSV, MCM-48-RSV-Physical mixture (PM) and MCM-48-RSV ( $n=3 \pm S . D$., $P$ value was determined by one-way ANOVA, $\left.{ }^{* * *} \mathrm{P}<0.0001\right)$. B) In vitro release of pure RSV and RSV loaded in MCM-48 in phosphate buffer saline (PBS) $(n=3 \pm$ S.D.). Adapted with permission from ref. 53. Copyright 2016, Elsevier.

In gels from DGG loaded with RSV nanosuspensions, RSV exhibited significantly faster profiles (100\% in 6 h) than coarse suspension in situ gels (50\% at the same time). The authors explained these results regarding the small diameter particle size of the nanosuspension resulting in a higher dissolution rate. To understand the RSV release mechanism, different mathematical models were fitted. The results established that RSV release from gel nanosuspensions was diffusion-controlled according to the Higuchi model, while the release from gel coarse suspension corresponded to Zero order kinetic model, indicating concentration-independent drug release..$^{60,}$

The major enhancement in the RSV release was a consequence of its improved solubility due to the encapsulation. The SPI nanoparticles caused the higher increase being the RSV release almost 4 times higher compared to free from. GPE-DP formulation also showed a good performance and in this case the enhancement was ca. 3 times.

\subsection{Penetration and permeation studies}


In general, the formulations proposed enhanced the penetration and permeation of RSV in comparison with a control solution. For instance, HA coated nanoemulsions confirmed the skin penetration of RSV through ex vivo sheep nasal mucosal and showed a RSV flux of $2.86 \mu \mathrm{g} / \mathrm{cm}^{2} \mathrm{~h} \cdot .^{25}$

In vitro permeation analysis measured in porcine skin at $37{ }^{\circ} \mathrm{C}$ showed that only $21 \%$ of RSV was penetrated from ethanol solution, whereas $82 \%$ was released from RSV-emulsion (with 2-HP- $\beta-C D$ ) evidencing its good skin compatibility (Figure 22). ${ }^{26}$

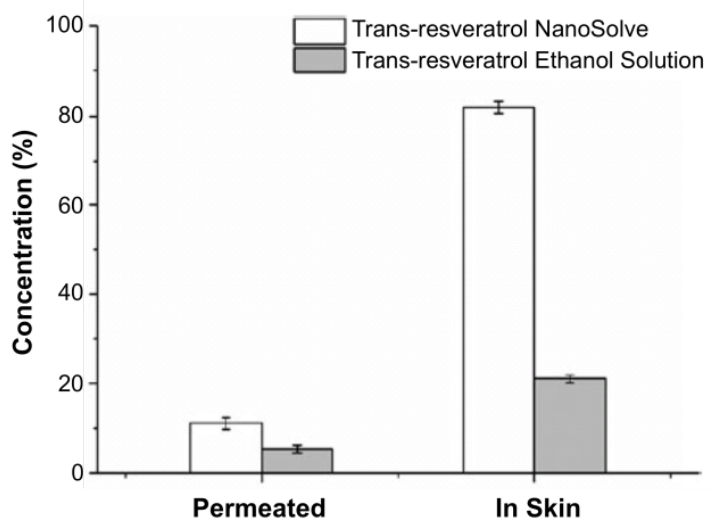

Figure 22. In vitro permeation results of trans-RSV ethanol solution and trans-RSV loaded in an emulsion with 2-HP- $\beta$-CD (NanoSolve). Adapted with permission from ref. 26. Copyright 2016, Taylor \& Francis.

It is worth mentioning that a higher cumulative amount of RSV-SNEDDS (composed of sodium lignin sulfonate/Tween 80/PEG-400 as mixed surfactants and olive oil) was transported, from the apical-to-basolateral direction through Caco-2 cells monolayers, than from pure RSV solution..$^{33_{1}}$

Similarly, the permeability of the encapsulated RSV in MCM-48 nanospheres across tight junctions of Caco-2 monolayers was more than 5 times higher than for RSV suspension. ${ }^{54}$

RSV permeation through dorsal skin of Wistar rats was found to be superior using a niosomal hydrogel (88 $\%$ vs. $63 \%$ for the plan drug suspension). Moreover, the skin retention for the niosomal gel was found to be 4.76 $\pm 0.21 \mathrm{mg} / \mathrm{cm}^{2}$, which corresponded to almost five times the value observed with plain drug suspension. Dermatokinetic studies confirmed the high RSV permeability in dermis and epidermis and the increase of its biological half-life using niosomal hydrogel..$^{57}$

In some cases, the improvement of RSV penetration and permeation were performed by reducing the size of particles or using penetration enhancers. In this sense, ex vivo penetration and permeation studies of RSV loaded niosomes were carried out using skin of newborn pig due to its similarity to the human skin. The authors found that the formulations prepared with EIM were more effective into dermis and epidermis layers, reaching 21 $\%$ RSV penetration with surfactant/fatty acid 1:1 ratio, whereas the formulations prepared with TFH-sonication only reached $11 \%$ at the same weight ratio (Figure 23). This could be due to the smaller size obtained using EIM, independently of the type of fatty acid used. Besides, it was observed that \% RSV penetration was not dependent of the RSV EE..$^{16}$

In vitro penetration experiments were performed with a nanosuspension (nanocrystals) using porcine ear skin via tape stripping. The results showed that after RSV application such as nanosuspension form, the drug was found up to the twentieth strip of the skin while from coarse suspension only penetrated until the tenth strip. $^{[56]}$

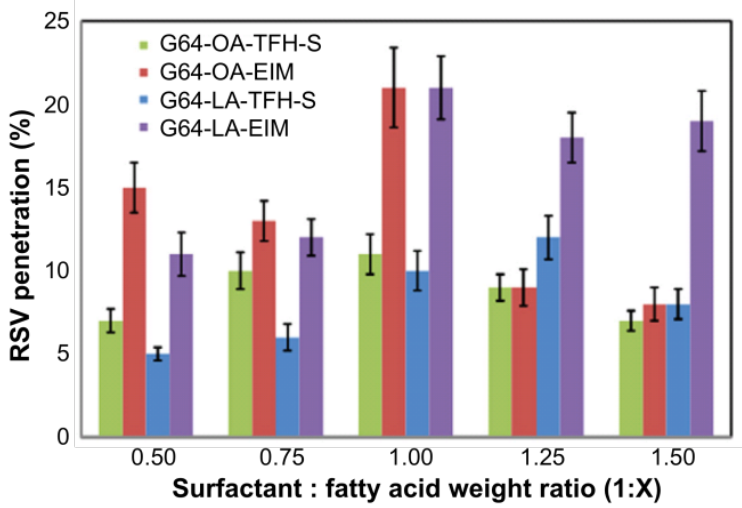


Figure 23. RSV penetration in epidermis and dermis (EDD): influence of formulation and niosomes preparation method. Niosomes made of Gelot 64 and Oleic Acid. TFH-S, thin film hydration combined with sonication method. EIM = Ethanol injection method. Adapted with permission from ref. 16. Copyright 2015, Elsevier.

Skin diffusion studies of soy phosphatidylcholine and OA (penetration enhancer) liposomes were also carried out on newborn pig skin. The results obtained were compared with conventional liposomes (without penetration enhancers). RSV accumulation in the whole skin increased 1.7 times with OrCG-liposomes and 2 times for LauFCC-liposomes. In the epidermis, RSV accumulation increased 1.3 and 2.5 times for OrCG and LauFCC-liposomes, respectively (Figure 24). Confocal imaging of the skin showed that the ultrastructure of the epidermis untreated appeared compact and thin, whereas the skin treated with the liposomal formulation became swollen and more fluid with loose structures, but without disrupting the integrity of the skin. Besides, a significant degree of skin hydration was also observed, which enhances its permeability and produces faster cutaneous diffusion of the drug by the vesicles. ${ }^{20}$

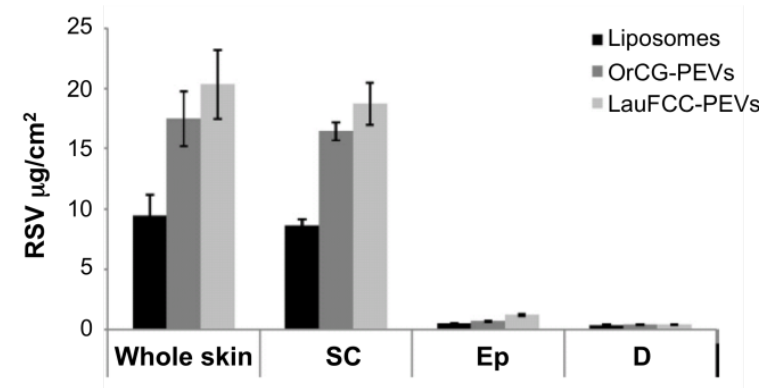

Figure 24. RSV deposition into pig skin layers (SC, stratum corneum; Ep, epidermis; D, dermis) and whole skin after $8 \mathrm{~h}$ non-occlusive treatment with RSV loaded in liposomes containing OrCG and LauFCC (penetration enhancers). Each value is the mean \pm S.D. of six experimental determinations. Adapted with permission from ref. 20. Copyright 2015, Elsevier.

In a similar way, nanoemulsions significantly increased intestinal permeation of RSV across rat jejunum. The RSV fluxes in the mucosal to serosal direction increased 6 times for semi-solid NE (nanoemulsions of Gelucire ${ }^{\circledR}$ 44-14 and Labrasol) and 8 times for liquid-NE (nanoemulsion from Miglyol ${ }^{\circledR} 812$ and Tween 80 ), compared to the control ethanolic RSV solution. Such significant difference was attributed to the nature of the formulations and to the droplet size difference between both nanoemulsions. ${ }^{29}$

Other permeation studies helped to understand the RSV metabolism when it is encapsulated. In the case of Labrasol-nanoemulsions, RSV metabolism was studied determining the permeation and the transport from serosal to mucosal layer (transfer rate) of RSV in rat small intestine. The results showed a 3.57-fold increase in transfer rate with Labrasol-nanoemulsions compared to free RSV. The molar ratio between permeated RSV-3glucuronide (RSV-G, metabolite) and RSV using free RSV and Labrasol-nanoemulsions was $7 \pm 2$ and $1.3 \pm 0.1$ respectively, indicating an inhibition of RSV glucoronidation due to encapsulation in nanoemulsions. ${ }^{24}$,

Furthermore, using SMEDDS (composed of Labrafil, Labrasol and Cremophor RH 40) in comparison with free RSV, the transport of RSV across Caco-2 cell monolayers was significant increased, ca. 4 times, indicating an improvement in RSV oral bioavailability. Besides, results from everted sacs of rats guts showed that RSV glucoronidation was notably inhibited when SMEDDS was used, since the molar ratio between RSV-G/RSV was $0.36 \pm 0.10$ against $7.25 \pm 0.48$ observed for free drug..$^{30}$

In other examples, coating material improved the permeation of RSV from the formulation. In vivo skin penetration studies were carried out in volar forearm of human volunteers free of dermatological disorders using the tape striping technique ${ }^{62,63}$. The results indicated that the fraction of RSV penetrated in the upper portion of the stratum corneum was ca. $49 \%$ for chitosan-coated microparticles and ca. $33 \%$ for uncoated ones. The former formulation also showed a marked and statistically significant increase of the fraction of RSV penetrated compared to the control formulation $(29 \%)$ in the same skin layers..$^{39}$

From solid lipidic nanoparticles (containing cetylpalmitate and Tween 80) functionalized with ApoE, permeability showed a significant increase (ca. 2 times higher) in penetration through hCMEC/D3 cells monolayers compared to non-functionalized ones. ${ }^{45_{1}}$

When co-encapsulation was employed using lipid-core nanocapsules for delivery of RSV and CUR, skin penetration experiments showed that in stratum corneum the amount of RSV was ca. 3 times higher when a drug solution was used instead of RSV-CUR-nanocapsules. However, in epidermis and dermis higher amounts of RSV were observed from RSV-CUR-nanocapsules. The penetration behavior of RSV from RSV-CURnanocapsules was attributed, with the aid of FT-IR, to the interaction of CUR with stratum corneum bilayers that facilitate RSV penetration in inner skin layers. ${ }^{52,}$

In summary, the best results in penetration and permeation were found with nanoemulsions, such as semi solids and liquids, due to their composition and size. In contrast, vesicles slightly increase these RSV properties. 


\subsection{Bioavailability}

The bioavailability of RSV was studied after administration via oral, intranasal or intravenous using different pharmacokinetic studies.

After oral administration, the bioavailability of RSV encapsulated in Labrasol-nanoemulsions was evaluated using male Winstar rats. When Labrasol-nanoemulsions were administered, it was found a peak concentration $\left(\mathrm{C}_{\max }\right)$ of RSV in rat plasma, ca. 13-fold higher than free RSV. Moreover, the area under concentration-time curve (AUC) of RSV with Labrasol-nanoemulsions was ca. 6 times higher that with free RSV. The ratio between $A U C_{R S V-G}$ and $A U C_{R S V}$ indicated an inhibitory effect of resveratrol-3-glucuronide (RSV-G) production due to Labrasol presence, since the ratio treated with Labrasol-nanoemulsions was 0.88 , a value considerable lower than that obtained with free RSV (10.12). ${ }^{24}$,

A remarkable impact of the formulation on the RSV metabolization pathways was also observed. In fact, in the permeation of RSV from an ethanolic solution through the serosal compartment, two main metabolites were observed. In contrast, these compounds were not observed when SNEDDS formulations were used. ${ }^{29}$

Pharmacokinetic studies revealed that the oral bioavailability of optimized RSV-SNEDDS increased ca. 3 times compared with unformulated RSV solution, which was attributed to the increase in RSV solubility and the small particle size of SNEDDS..$^{28}$

Additionally, the biodistributions of RSV in the mesenteric lymph were measured after oral administration. SMEDDS formulations (composed of Labrafil, Labrasol and Cremophor RH 40) significantly improved the distribution of RSV in the intestinal lymph nodes compared with free drug at all time-points. Finally, the results obtained from in vivo pharmacokinetic study showed a RSV absolute bioavailability of 76 \% (free RSV group revealed merely $6.5 \%$ ). Besides, the AUC RSV-G $_{\text {G }}$ decreased significantly by $73 \%$ compared with free RSV. ${ }^{30}$

Various pharmacokinetic parameters revealed directly the enhanced adsorption rate of RSV SNEDDS. The AUC of the proposed formulation resulted in a 72-fold increase in bioavailability and $C_{\max }$ value rose considerably to 24 fold as compared to RSV suspended in water. ${ }^{33}$ Similarly, in vivo studies revealed a 5 -fold increase of the bioavailability of RSV encapsulated in solid lipidic nanoparticles compared to RSV suspension. Furthermore, pharmacodynamic experiments showed a significant decrease in the serum biomarker enzymes (serum glutamic oxaloacetic transminase, serum glutamate pyruvate transminase and alkaline phosphatase) after oral administration of the proposed formulation in comparison with the control and marketed formulations against paracetamol-induced liver cirrhosis. ${ }^{44}$

In the case of TMC-g-palmitic acid SLNs, pharmacokinetics assays were performed in mice after oral dose of the formulation. The results showed that in that condition RSV oral bioavailability increased ca. 4 fold in comparison with SLNs. The authors describe this improvement as a result of the mucoadhesive and absorption properties of the polymer, which protects RSV from acidic and enzymatic gastric conditions. ${ }^{43}$

Results obtained from other studies indicate that when GPE-DP formulation was used instead of GPE formulation, the RSV absorption via oral was raised ca. 12 times. ${ }^{46}$

In addition, in vitro digestion tests were carried out to study the digestive stability and bioaccessibility of trans-RSV simulating oral, gastric and intestinal phases. RSV digestive stability was $81 \%$ and $47 \%$ for sodium caseinate microparticles and unencapsulated RSV, respectively. Moreover, the RSV bioaccessibility after 3phase in vitro digestion from sodium caseinate microcapsules was 2.56 fold higher than the control (59\% vs. 23 $\%$, respectively). ${ }^{47}$ Moreover, the bioaccesibility of RSV was improved significantly when core-shell nanoparticles were mixed with lipid droplets (forming nanoemulsions), (i.e. $43 \%$ in absence and $90 \%$ in the presence of nanoemulsions). ${ }^{49}$

Regarding other pharmacokinetic studies, $\mathrm{AUC}_{0 .-}$ and $\mathrm{C}_{\max }$ of RSV were increased by 3.5- and 2.2-fold, respectively, when nanocrystals was used instead of plain RSV. Furthermore, mean residence time was raised ca. 1.2 times for RSV-nanocrystals (Figure 25). Such upgrading on RSV bioavailability could be due to several factors, such as the improved dissolution and reduced thickness of diffusion layer due to the small size, the increased bioadhesion of particles to the intestinal epithelium and the direct uptake of particles across the intestinal barrier. ${ }^{55}$ 


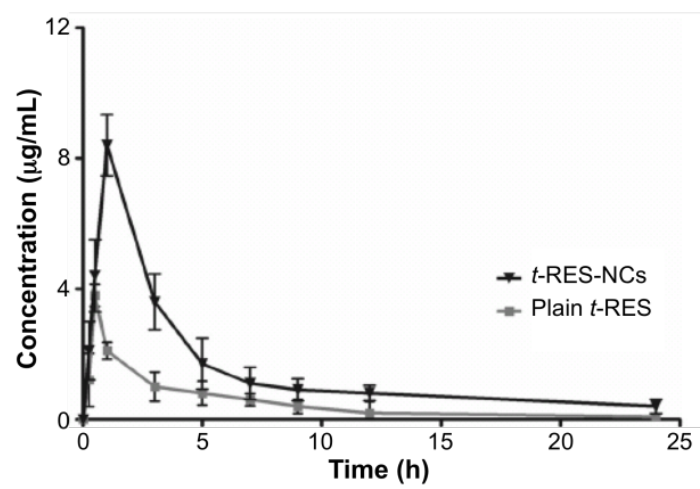

Figure 25. Plasma concentration time profiles of RSV after oral administration of plain trans-RSV and trans-RSV-nanocapsules (NCs) to $\mathrm{SD}$ rats at $20 \mathrm{mg} / \mathrm{kg}$ dose. Values are expressed as the mean \pm S.D. $(\mathrm{n}=6)$. Adapted with permission from ref. 55. Copyright 2017, Springer.

Otherwise, the RSV bioavailability was analyzed through nasal mucosa and the in vivo quantification in the brain of male Albino rats, showed ca. 7-fold increase in $\mathrm{AUC}_{0-7 \mathrm{~h}}$ when the nanoemulsion formulation was used instead of RSV free solution. ${ }^{25}$ A drastic increase was observed in RSV uptake in the cerebrospinal fluid of rats using lipidic particles coated with chitosan $(8.75 \% \mathrm{v} / \mathrm{w})$ in comparison with uncoated particles and pure RSV. Indeed, the obtained RSV $\mathrm{C}_{\max }$ after 60 min of nasal administration was 12.2-fold higher than for uncoated particles while no detectable amounts was noticed from pure RSV. ${ }^{[4]}$

The bioavailability of RSV was also performed using gel nanosuspensions administered via intranasal and intravenous routes. The results showed that the bioavailability in brain was enhanced ca. 3 times in the case of intranasal administration instead of intravenous. Moreover, the brain drug direct transport \% demonstrated that approximately $78 \%$ of RSV was absorbed into the brain directly from the nasal cavity, while RSV into the brain was not detected using intravenous route..$^{60}$

In general, RSV bioavailability is a property scarcely studied. However, nanoemulsions were the delivery system that showed the best performance, either after oral or nasal administration.

\subsection{Stability against photoisomerization}

The stability of trans-RSV was studied after light exposure. For example, the isomer degradation in the SNEDDS was evaluated inside and outside the dialysis bag. Interestingly, the results showed that SNEDDS greatly reduced the photochemical degradation of trans-RSV, whereas the amount released and degraded of RSV from free solution was 50-50, which may be due to the directly contact with light and oxygen. ${ }^{33}$,

After direct UV irradiation the antioxidant activity of free RSV significantly decreased, but RSV in nanoparticles coated with chitosan still kept high activity, demonstrating than they could protect RSV from light destruction and increased its bioavailability. ${ }^{41}$

On the other hand, UV measurements performed using UVA light of $365 \mathrm{~nm}$ for $1 \mathrm{~h}$ showed a trans/cis RSV ratio of 0.65 for microparticles of sodium caseinate and 0.49 for unencapsulated RSV. ${ }^{47}$, Moreover, when the samples were exposed to UV-light for $1 \mathrm{~h}$, the retention of RSV free solution was $65 \%$, while the retention of RSV trapped in nanoparticles was $90 \%$. This effect was attributed to the presence of the nanoparticles, which decreased the amount of UV-light reaching the RSV due to light scattering or absorption effects. ${ }^{49}$. Dai and coworkers also studied the structural stability of encapsulated RSV in lignin nanoparticles after $1 \mathrm{~h}$ of direct sun light. They observed only a decreased of $2 \%$ of trans to cis RSV. ${ }^{[51]}$

RSV-CD complex encapsulated in phospholipid nanoemulsions also prevented the degradation of RSV after UV irradiation (365 nm) (i.e. degradation \% of RSV in water/ethanol was 64, where it was 25 within CDnanoemulsions) (Figure 26). Although these nanoemulsions did not prevent completely the RSV degradation, they slowed down its rate. The formulation offers two protective layers (i.e. CD and nanoemulsions), which shielded RSV from UV light. ${ }^{59}$ 


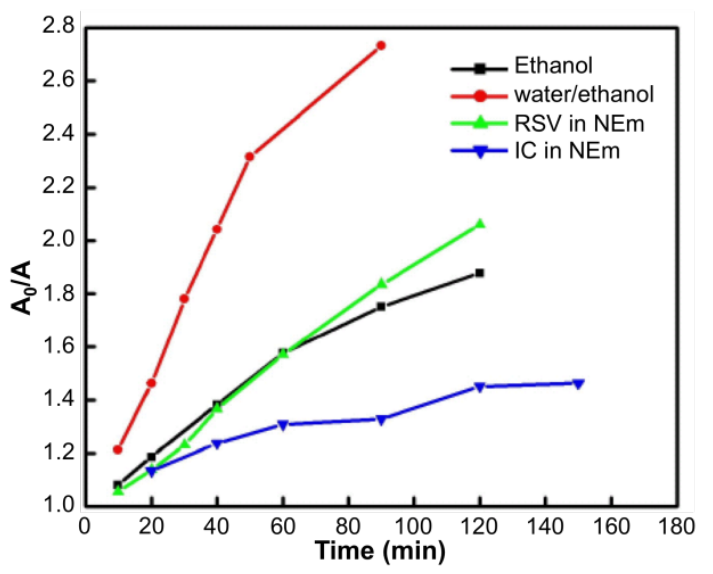

Figure 26. Absorbance reduction intensity plots of RSV in different conditions after UV irradiation (365 nm). NEm (nanoemulsions) and IC (inclusion cyclodextrin complex). Adapted with permission from ref. 59. Copyright 2017, Elsevier.

In summary, even though the instability of bioactive RSV after light exposure is an important issue to be studied in a proposed formulation, just a few papers have provided such results. From those, core-shell nanoparticles mixed with lipid droplets retained almost the complete trans-RSV after $1 \mathrm{~h}$ of UV-light exposure.

\subsection{Biocompatibility}

Regarding vesicles, niosomes formed by Span 60 and cetyl alcohol presented very low or negligible toxicity against RAW264.7 macrophage cells (i.e. $92.5 \%$ of cell viability at $40 \mu \mathrm{M}$ )[19]. Similarly, liposomes formed by soy phosphatidylcholine and OA indicated non-cytotoxicity using 3 T3 fibroblasts. ${ }^{20,}$

A different liposomal formulation (PEG-modified liposomes), was incubated with human erythrocytes and no significant effect was observed, whereas free RSV ethanolic solution caused human red blood cells hemolysis under the same conditions. ${ }^{22}$ Similar results were observed with the nanoemulsion coated with HA, that did not cause any tissue damage or epithelial and ciliotoxicity in the nasal mucosa. ${ }^{25}$

Additionally, macrophage uptake and imaging studies showed that the nanoemulsions were fully internalized by the macrophage cell line, RAW 264.7 cells. Importantly, the nanoemulsions did not affect macrophage viability of lipopolysaccharide-stimulated macrophages at the highest dose and decreased the NO production indicating no oxidative processes in the cells. ${ }^{27_{1}}$

In another study of the cytotoxicity of SMEDDS (composed of Labrafil, Labrasol and Cremophor RH 40 as oil phase), the results indicated no increase of toxicity towards Caco-2 cells compared with free RSV. ${ }^{30,}$

Concerning the cell/tissue integrity and viability of SNEDDS (sodium lignin sulfonate/Tween 80/PEG-400 and olive oil), the authors reported that the system exhibited relatively lower toxicity than a pure RSV solution..$^{33_{1}}$

In vitro cell viability studies of solid lipid nanoparticles modified with a chitosan derivative were performed using NIH/3T3 mouse embryonic fibroblast cells and none of the formulations showed any significant toxicity. ${ }^{43_{1}}$ Moreover, in vitro toxicity studies of solid lipid nanoparticles functionalized with ApoE revealed no toxicity up to $50 \mu \mathrm{M}$ over $4 \mathrm{~h}$ of incubation using hCMEC/D3 cells. ${ }^{45}$

In contrast, cell viability studies of lipid-core nanocapsules using human dermal fibroblasts showed a significant alteration of the profile compared to the control cells $(80 \%) .^{52}$

Furthermore, cell viability test was performed with Caco-2 and RAW 264 cells, indicating no significant toxicity of pristine MCM-48 nanospheres..$^{54}$

Finally, in vitro hemolysis test of the multicompartamental nanovehicle based on casein micelles and phytosomes showed low or negligible hemolytic activity $(<5 \%)$ indicating a safely parenteral delivery of the nanocarrier..$^{61}$

In general, most of the mentioned delivery systems showed negligible toxicity against different types of cells and they were found to be safe RSV carriers.

\section{Potential applications}

\subsection{Pharmaceutical perspective}

\subsubsection{Antioxidant activity}

As it is crucial to verify that the vesicle system does not alter RSV performance, in vitro antioxidant activity of RSV encapsulated in different systems was performed using 2,2-diphenyl-1-picrylhydrazyl (DPPH) radical assays. 
No significant difference in scavenging activity was observed between free and encapsulated RSV in niosomas (Span 60 and cetyl alcohol) at the same concentration, confirming the retention of RSV biological functionality after its encapsulation into the vesicles. Besides, assays in macrophage RAW264.7 cell lines showed that niosomes have higher intracellular reactive oxygen species scavenging activity than those of RSV in its free form. Regarding this intracellular scavenging activity, lipopolysaccharide-stimulated macrophages showed a considerably reduction of fluorescence degree when RSV niosomes where used instead of free RSV. Thus, nanoencapsulation enhanced the functional property of RSV, possibly due to its higher degree of cell internalization. ${ }^{19}$

The results of in vitro antioxidant activity assays of RSV in liposomas showed that the liposomal formulation did not affect the antioxidant activity of the polyphenol $(93 \pm 1 \%)$, regardless the presence of PEG-surfactants. In addition, the antioxidant activity of RSV was assessed ex vivo in human erythrocytes by cellular antioxidant activity test, which revealed a dose-dependent scavenging effect of RSV PEG-modified liposomes on oxidative stress induced by peroxyl radical generator on human red blood cells, regardless the concentration..$^{22}$

In addition, the $\mathrm{pH}$ of lipidic nanoemulsions developed by Nasr ranged from $6.6-6.7$ and the antioxidant assay demonstrated no statistical differences in the percentage of radical inhibition upon RSV encapsulation..$^{25}$,

It is very interesting to note that the antioxidant activity of multilayer (lactoferrin/alginate and lactoferrin/alginate/ $\varepsilon$-poly-L-lysine) emulsions containing RSV, did not present significant changes during storage using DPPH radical scavenging activity assay for 4 weeks. ${ }^{37}$,

In a similar way, the antioxidant activity of RSV could be maintained and enhanced after encapsulation into chitosan coated nanoparticles since the DPPH scavenging capacity was increased $10-30 \%$ for RSVnanoparticles in comparison with free RSV, which was attributed to the own activity of chitosan..$^{41}$

Besides, free DPPH radical scavenging activity was determined, showing $\mathrm{IC}_{50}$ values of $62 \mu \mathrm{M}$ and $65 \mu \mathrm{M}$ for RSV-nanoemulsions and RSV-CD-nanoemulsions, respectively. RSV-CD-nanoemulsions displayed a slightly higher $\mathrm{IC}_{50}$ value, which could be ascribed to the insertion of RSV molecules into the hydrophobic core of CD, which acted as a barrier between RSV and DPPH radical. ${ }^{59}$ Furthermore, the antioxidant activity of core-shell nanoparticles was determined using 2,2'-azino-bis(3-ethylbenzothiazoline-6-sulphonic acid (ABTS) free radical scavenging activity assay. The nanoparticles containing both polyphenols (RSV and CUR) exhibited higher antioxidant activity than single loaded nanoparticles, as expected..$^{49}$

Free radical scavenging assays with RSV-loaded chitosan nanoparticles, exhibited a $\mathrm{IC}_{50}$ of $19 \mu \mathrm{g} / \mathrm{mL}$ with DPPH, a $5 \mu \mathrm{g} / \mathrm{mL}$ with ABTS and $0.161 \mathrm{mg}$ ferrous sulfate/1 mg de RSV-lipidic nanoparticles with ferric reducing antioxidant power. These results indicated a strong protective effect of the formulation against cell oxidative stress damage. ${ }^{41}$

A synergic effect in the antioxidant activity between CUR and RSV was observed through co-encapsulation in hyalurosomes made of a synthetic polymer. In this work, the percent of DPPH inhibition was 4-fold higher for CUR-RSV-hyalurosomes than for single loaded ones. ${ }^{[50]}$

Furthermore, none of the studied systems modified the antioxidant capacity of RSV.

\subsubsection{Anticancer activity}

The RSV anticancer activity in the works presented here was studied in liver, colon, breast and lung cancer cells. In an interesting study with very good prospective, lipidic nanostructures containing RSV showed a significant inhibition in human liver tumor cells (HepG2 cell) growth. ${ }^{42_{1}}$

In comparison, RSV in chitosan nanoparticles and free RSV presented equivalent human hepatocellular carcinoma cells and human normal hepatocyte cells cytotoxicity, at the same concentration. Furthermore, for normal cells, RSV encapsulated and pure RSV showed lower cytotoxicity in comparison with Cis-Pt at above 20 $\mu \mathrm{g} / \mathrm{mL} .^{41_{1}}$

Other anti-cancer activity studies were performed in colon cancer cell lines, suggested that RSV in MCM48 was as effective as RSV free solution. However, nanoparticles substantially decreased intracellular apoptosis protein I expression, while control solution showed little impact on this process. ${ }^{53}$ Additionally, dual encapsulated liposomes (RSV-CD inclusion complex-liposomes) showed better anti-proliferative properties compared to free RSV, owing to endocytosis and faster release of RSV from this formulation. ${ }^{58,}$

Cytotoxicity experiments were carried out in breast cancer cells (MDA-MB 231 cells) and the half maximal inhibitory concentration $\left(\mathrm{IC}_{50}\right)$ for RSV-nanocrystals was 1.25-fold higher in comparison to plain RSV within $24 \mathrm{~h}$, which could be due to the direct uptake or increased dissolution of RSV-nanocrystals. ${ }^{55_{1}}$

When micellar casein nanoformulations were used, in vivo assay was performed in breast tumor induced in animal groups, at the dose of $5 \mathrm{mg} / \mathrm{kg}$ per day of RSV up to 3 weeks along with free RSV. The tumor volume observed after treatment with free RSV and the formulation was $882.6 \mathrm{~mm}^{3}$ and $60.2 \mathrm{~mm}^{3}$, respectively (the untreated positive control group had a tumor volume of $1433.3 \mathrm{~mm}^{3}$ ) suggesting excellent antioneoplasic effect of the micellar nanosystem. Two tumor growth biomarkers (aromatase and factor Kappa-B) were analyzed in order to evaluate the in vivo antitumor efficacy of the proposed formulation. In this regard, a significant reduction of the aromatase biomarker was recorded for the developed nanocarrier (15.43 $\mathrm{ng} / \mathrm{mg}$ tissue protein) in comparison with free RSV and with the untreated positive control group (50.85 vs. $77.14 \mathrm{ng} / \mathrm{mg}$ tissue protein). In 
addition, a significant inhibition of nuclear factor Kappa-B factor (NF- $\kappa B$ ) was observed in the micelles-treated group, compared with free RSV and untreated group (1.71, 7.82 and $10.10 \mathrm{ng} / \mathrm{mg}$ tissue protein, respectively). Moreover, a marker of apoptosis, caspase-3, was also evaluated as well, showing that micelles treated group exhibited 2.4 times higher caspase-3 level and higher tumor necrosis compared with free RSV..$^{61}$

The in vivo anticancer activity of lignin magnetic nanoparticles was studied in Lewis lung carcinoma tumorbearing mice. The results showed a significant inhibition in the tumor growth compared with RSV alone $(82.2 \%$ vs. $21.2 \%$, respectively) and the nanocarrier improved the survival rates on the experimental period being 83.3 $\%$ against $33.3 \%$ for RSV control. These facts were attributed to a RSV sustained release, a small particle size and good magnetic properties. ${ }^{[51]}$

As a general conclusion, the anticancer performance of RSV in the different nanoencapsulation systems tested was improved in comparison with RSV in plain solutions. The selection of the better system for each application will be related to the required form of administration.

\subsubsection{Anti-inflammatory activity}

In vitro NF-KB factor inhibition assay was performed measuring the green fluorescent protein expression using macrophage NF-KB reporter cells. The results revealed that encapsulated RSV in SPI nanoparticles was stable and its anti-inflammatory bioactivity was retained since non-significant modification in the green fluorescent protein reduction was obtained as compared to free drug. ${ }^{48}$

Anti-inflammatory studies of RSV in MCM-48 formulation on lipopolysaccharide-activated RAW 364.7 cells, showed a significant down regulation of NF-KB factor expression in comparison with RSV free solution demonstrating improved anti-inflammatory activity. ${ }^{53_{1}}$

Moreover, the in vitro NF-kB assay showed that RSV encapsulation in MCM-48 did not alter its bioactivity and, at lower concentration of $5 \mu \mathrm{g} / \mathrm{mL}$, RSV encapsulation provided higher anti-inflammatory activity compared to both RSV suspension and solution..$^{54}$

Finally, in vivo pharmacodynamic study showed that the percentage of inhibition in paw oedema from RSV encapsulated in niosomal hydrogel, was comparable with a commercial formulation (diclofenac Sodium), albeit the former displayed a prolonged therapeutic action..$^{57}$

It should be noted that the nanoencapsulation of RSV in the different systems analyzed did not reduce its anti-inflammatory activity, but it was improved in some cases.

\subsubsection{Physical fatigue}

It has been previously demonstrated that supplementation with certain antioxidant nutrients could be a practical approach for rapid recovery from fatigue and for preventing exercise-induced oxidative damage ${ }^{64}{ }^{6}$ Thus, Yen and co-workers performed a pretreatment to rats with a single dose of the RSV-SNEDDS before swimming exercise. The effectiveness of the formulation was followed by lactate production and clearance test, and blood biochemical variables (i.e. plasma ammonia, creatinine phosphokinase and glucose). In this study, the lactate production did not differ significantly compared with RSV solution albeit the pretreatment using RSV-SNEDDS (Capryol $^{\mathrm{TM}}$ 90, Cremophor ${ }^{\circledR} \mathrm{EL}$ and Tween $20(60: 35: 5 \% \mathrm{w} / \mathrm{w} / \mathrm{w})$ before exercise accelerated the recovery of lactate after exercise (ca. 1.4 fold faster). Concerning biochemical variables, the plasma ammonia level decreased significantly (by $35 \%$ ) compared with RSV solution associated to less peripherical and central fatigue. ${ }^{65}$ However, the plasma creatinine phosphokinase and the glucose levels showed no differences with respect to RSV solution.

Exhaustive swimming test showed that RSV-SNEDDS increased the swimming time to exhaustion of rats ca. 2 times compared with RSV solution, confirming that the formulation proposed improved not only the RSV bioavailability but also the exercise performance of rats. In addition, the formulation positively modulated exercise-induced fatigue-related parameters. ${ }^{28}$

\subsection{Food perspective}

\subsubsection{Food enrichment}

Textural analysis (firmness and adhesiveness parameters) of yoghurt enriched with RSV niosomes was performed, comparing the results with regular yoghurt as control. RSV Span 60-Dod showed similar firmness values compared to the control (i.e. $7.6 \pm 0.1 \mathrm{gF}$ vs. $7.5 \pm 0.3 \mathrm{gF}$, respectively). In addition, RSV Mai-Dod showed adhesiveness very close to control yoghurt (i.e. $-4.17 \pm 0.05 \mathrm{gF}$ vs. $-4.0 \pm 0.5 \mathrm{gF}$, respectively). ${ }^{18}$

\section{Preparation methods}

In this section, a brief description of the main preparation methods mentioned in this review is given. For the sake of clarity, the methods are classified depending on the type of confining material that is formed. 


\subsection{Vesicles}

The thin film hydration (TFH) is a simple method and widely used to produce vesicles as liposomes, niosomes, among others. In this method, the membrane components (non-ionic surfactants, phospholipids) are solubilized in an organic solvent. Then, a thin film is formed by completely removing the organic solvent under vacuum. After that, the dried film is hydrated above the transition temperature of the amphiphilic compound, using an aqueous solution (buffer solution, water) to obtain multilamellar vesicles..$^{66}$ In general, further methods are used to reduce the size/lamellae of the vesicles such as sonication. ${ }^{17,18,20}$ extrusion (five times through a $100 \mathrm{~nm}$ membrane), ${ }^{58_{1}}$ etc.

In the direct sonication method the suspension composed by the membrane components and the aqueous phase is sonicated in an ice bath during several short cycles of high intensity, using a probe sonicator, ${ }^{19,21,22}$

In the ethanol injection method (EIM), the membrane components are dissolved in ethanol and injected slowly through a needle in an aqueous solution maintained at constant temperature. Then, the organic solvent is evaporated and during this process is leaded to the formation of single layered vesicles. ${ }^{67}$

\subsection{Emulsions}

\subsubsection{Single emulsions}

Single emulsions can be prepared using simple methods. Fang and co-workers prepared a transparent microemulsion using high-pressure homogenization method whereby the resulting pre-emulsion is passed through a homogenizator at a pressure of 800 bar up to 4 cycles and at $80{ }^{\circ} \mathrm{C} .{ }^{68}$ Matos and co-workers prepared microemulsions by high shear homogenization method using high agitation rate in short time (20000 rpm for 1 $\min ) .^{32}$

In 2017, Kumar and co-workers prepared nanoemulsions using ultrasonic emulsification technology. ${ }^{59}$, This technique is more efficient than other mechanical techniques in terms of production cost, handling and maintenance..$^{69}$ The nanoemulsion is formed in two steps, firstly the formation of primary droplets of the suspension, and then the breakup of them by impact of cavitation forces induced by acoustic waves..$^{70,71}$

Besides, nanoemulsions can be prepared using self-assembly emulsification method, which provides ultralow interfacial tensions and large O/W interfacial areas, and thus, have high solubilization capacity and thermodynamic stability. The method is accomplished using an isotropic mixture of oil, surfactant, and cosurfactant to form fine $\mathrm{O} / \mathrm{W}$ emulsions upon introduction into aqueous media under agitation..$^{35}$

\subsubsection{Double emulsions}

Double emulsions can be prepared by conventional mechanical emulsification in two-steps. Thus, Matos and coworkers prepared the primary W/O emulsion in glass vessels by high shear mixing at $15000 \mathrm{rpm}$ for $10 \mathrm{~min}$. In the next step, the primary emulsion was dispersed into the external aqueous phase $\left(\mathrm{W}_{2}\right)$ at $5000 \mathrm{rpm}$ for 2 min to form $\mathrm{W}_{1} / \mathrm{O} / \mathrm{W}_{2}$ emulsion. ${ }^{38}$

The membrane emulsification is another technology used to form double emulsions that consists in a dropby-drop emulsification through a microporous membrane. In addition, this method allows the production of uniform particles with controlled droplets size, low shear stress and energy requirement. ${ }^{72}$ Matos and co-workers produced food-grade double emulsion using tubular membranes in the cross-flow system and a glass membrane with a nominal $10 \mu \mathrm{m}$-pore diameter. ${ }^{36_{1}}$ In this experimental design (Figure 27), the continuous phase flows through the membrane internal channel, and oil droplets are detached from the membrane surface forming the emulsion, which is recirculated to the continuous phase reservoir. The continuous and dispersed phases are pumped at a controlled flow rate. 




Figure 27. Schematic diagram of the A) membrane emulsification unit and B) the membrane module. Reproduced with permission from ref. 36. Copyright 2015, Elsevier.

\subsection{Particles}

\subsubsection{Lipidic particles}

Lipidic particles consist of a solid hydrophobic core stabilized by a hydrophilic layer of surfactant molecules or polymers, as was previously mentioned. The solid dispersion can be prepared by hot melt method, where the lipidic material is heated at or above the melting point and then cooled rapidly. This process leads to form fine solid particles by supersaturation..$^{73}$ In 2017 , Chang and co-workers obtained spherical particles of milimetrical size using this method. ${ }^{46}$ Scalia and co-workers prepared microparticles combining the hot-melt method with sonication (at $20 \mathrm{kHz}$ for $5 \mathrm{~min}$ ) and lyophilization, to obtain water-free microparticles. ${ }^{39}$ On the other hand, nanoparticles could be prepared using additionally high pressure homogenization, ${ }^{41_{1}}$ sonication ${ }^{44_{1}}$ or ultrasonication. ${ }^{42}$

Solid lipid based nanoparticles (SLNs) can also be prepared by solvent injection whereby the lipidic components are dissolved in ethanol at $40^{\circ} \mathrm{C}$ and injected into the aqueous phase containing the surfactant at the same temperature. The resulted dispersion is filtered, centrifugated and washed in deionized water. The recovered nanoparticles are re-suspended, frozen and freeze-dried to obtain the SLNs. ${ }^{43}$,

\subsubsection{Proteic particles}

Koga and co-workers fabricated microparticles made of sodium caseinate using the spray drying method. ${ }^{47}$ In general, in this method, the formulation components are dissolved into a solvent and the fine solid particles generated by atomizing the solution into a drying process..$^{73_{1}}$

In 2018, Liu and co-workers developed nanoparticles based on protein-polyphenol conjugates by the antisolvent precipitation method. Here, zein-epigallocatechin gallate:RSV were dissolved in ethanol and added in an aqueous solution containing the biosurfactant. Finally, the ethanol in the colloidal dispersion formed was removed using a rotary evaporator and the same volume of water was added to compensate the lost of organic solvent. ${ }^{49}$ A similar experimental procedure was performed by Pujara and co-workers to produce SPI-RSV complex nanoparticles. ${ }^{48}$

\subsection{Nanocapsules}

Lipid-core nanocapsules are a specific type of nanocapsules composed of an oily core surrounded by a polymeric shell stabilized by surfactant in aqueous medium. Friedrich and co-workers used the precipitation method of preformed polymer, where the oily core is dissolved in acetone at $40{ }^{\circ} \mathrm{C}$ and injected in the aqueous phase containing the surfactant under moderate stirring. Subsequently, the organic solvent is evaporated and the aqueous phase is concentrated under reduced pressure to a final volume..$^{52}$

\subsection{Mesoporous silica nanoparticles}


Summerlin and Juère prepared mesoporous silica nanoparticles based on Stöber method in presence of a surface-active molecule. In this bottom-up synthesis, the tetraethylorthosilicate is used as a silica source, the surfactant and the polymer act as structure-directing and dispersion agent, respectively. Briefly, the surfactant and the polymer are dissolved in a mixture of ethanol and ammonium hydroxide. Then, tetraethylorthosilicate is added and the mixture stirred. After that, the mixture is centrifugated and the solid is washed and dried at high temperatures and prolonged times. Finally, the resulting product is calcinated. ${ }^{53,54,}$

\subsection{Nanocrystals}

Singh and co-workers prepared $t$-RSV nanocrystals using probe sonication. Shortly, the drug is dispersed in an aqueous phase containing the stabilizers and stirred to homogenization. Further, the sample is sonicated for different time intervals in an ice bath. Then, the sample is pre-frozen and lyophilized. ${ }^{55}$

Sinico and co-workers used wet media milling technique to prepare RSV nanocrystals. ${ }^{[56]}$ Briefly, RSV dried powder is mixed with an aqueous solution containing stabilizers and subsequently, a mechanical energy is applied to physically break down coarse particles to smaller ones..$^{74}$ In the work of Sinico and co-workers, the authors used sieving to separate milling beads (composed by yttrium-stabilized zirconia-silica) from nanocrystals. ${ }^{[56]}$

\section{Summary and Outlook}

In this review, we have summarized the most representative delivery systems developed for RSV during the last 5 years. These carriers have been designed with the aim to overcome some of the limitations derived from the low aqueous solubility, intestinal permeation, bioavailability, and photostability of RSV. The developed delivery systems included liquid formulations involving e.g. vesicles, emulsions and nanocapsules, as well as solid formulations based on e.g. mesoporous silica nanoparticles and nanocrystals. Combinations of both types of systems have also been explored for nutritional and pharmaceutical applications.

In the case of RSV-containing vesicles, most studies have demonstrated that the mean size, distribution, encapsulation efficiency, and stability are determined by the preparation method rather than by the chemical composition of the vesicles. In terms of administration routes in the pharmaceutical field, the incorporation of penetration enhancers in vesicle-based formulations constitutes a common strategy for topical administration. In addition, RSV-containing liposomes have been explored for the treatment of different diseases, via oral administration, associated with inflammation and oxidative stress in tumour cells. Other carriers such as surfacemodified nanoparticles, vesicles-in-hydrogels, nanocapsules (for topical administration) and emulsions (for oral administration) containing inhibitory excipients have also been developed with the aims of enhancing the RSV bioavailability and antioxidant effects. Furthermore, dual encapsulation strategies such as the inclusion of cyclodextrins into liposomes or nanoemulsions allow an increase of the RSV loading, compared to conventional vesicles. In this case, surface modifications could be performed to upgrade the in vivo performance of the system, e.g. cell-specific targeting, cellular uptake or clearance. Another promising dual strategy is the incorporation of RSV-containing vesicles (e.g. niosomes) into hydrogel formulations, which increases the bioavailability of RSV and provides higher resistance and tuneable drug delivery due to the mechanical and responsive properties of the gel matrices.

The higher efficiency achieved with most of the aforementioned systems is related to the enhanced aqueous solubility of RSV. Other disadvantages of RSV such as low photostability have been addressed using biopolymer-based self-nanoemulsifying delivery systems.

Despite the growing number of publications in this area during the last years, and the promising applications of RSV-containing carriers in pharmaceutical and food industries, more research is still necessary for a better understanding of barrier penetration mechanisms of RSV, the impact of food matrices on the stability and controlled delivery of RSV, as well as the pharmacodynamics of the proposed formulations. In addition, those studies should also consider the necessity of preserving the structural integrity of the carriers in biological fluids while retaining the antioxidant capacity of RSV. In this context, the development of combined delivery systems and the generation of larger number of in vivo data should guide future research in this area.

\section{Conflicts of interest}

There are no conflicts of interest to declare.

\section{Acknowledgments}

This work was supported by the University of Regensburg. N.D.M. thanks Consejo Nacional de Investigaciones Científicas y Técnicas (CONICET) for the fellowship and the International PhD program (iPUR) at the University of Regensburg for the research internship granted. M.A.F. thanks Consejo Nacional de Investigaciones 
Científicas y Técnicas (CONICET, PIP 112-2015-01-00242), Agencia Nacional de Ciencia y Tecnología (FONCyT, PICT 2014-2516) and Universidad Nacional de Córdoba (SECyT-UNC 2016-2017) for the grants received. D.D.D. thanks the Deutsche Forschungsgemeinschaft (DFG) for the Heisenberg Professorship Award.

Keywords: drug delivery systems $\bullet$ encapsulation $\bullet$ nutrition $\bullet$ pharmaceutics $\bullet$ resveratrol

[1] J. Gambini, R. López-Grueso, G. Olaso-González, M. Inglés, K. Abdelazid, M. El Alami, V. Bonet-Costa, C. Borrás, J. Viña, Rev. Esp. Geriatr. Gerontol. 2013, 48, 79-88.

[2] D. Pando, G. Gutiérrez, J. Coca, C. Pazos, J. Food Eng. 2013, 117, 227-34.

[3] G. Davidov-Pardo, D. McClements, Trends Food Sci Technol. 2014, 38, 88-103.

[4] D. Arora, S. Jaglan, in Nanoscience in Food and Agriculture 5; (Eds.: S. Ranjan, S., N. Dasgupta, E. Lichtfouse), Springer Netherlands, 2017, pp 123.

[5] J. A. Baur, D. A. Sinclair, Nat. Rev. Drug Discov. 2006, 5, 493-506.

[6] M. A. Vian, V. Tomao, S. Gallet, P. O. Coulomb, J. M. Lacombe, Chromatogr. A 2005, 1085, $224-29$.

[7] G. Montsko, M. S. P. Nikfardjam, Z. Szabo, K. Boddi, T. Lorand, R. Ohmacht, L. Mark, J. Photochem. Photobiol. A Chem. 2008, 196, 44-50.

[8] M. M. Lyons, C. Yu, R. B. Toma, S. Y. Cho, W. Reiboldt, J. Lee, R. B. Van Breemen, J. Agric. Food Chem. 2003, 51, 5867-70.

[9] S. Zupančič, Z. Lavrič,J. Kristl, J. Eur. J. Pharm. Biopharm. 2015, 93, 196-204.

[10] J. Flieger, M. Tatarczak-Michalewska, E. Blicharska, Anal. Lett. 2017, 50, 294-303.

[11] M. Del Carmen Pinto, J. A. García-Barrado, P. Macías, J. Agric. Food Chem. 1999, 47, 4842-46.

[12] G. L. La Torre, G. Laganá, E. Belloco, F. Vilasi, F. Salvo, G. Dugo, Food Chem. 2004, 85, 25966.

[13] J. P. Gleeson, S. M. Ryan, D. J. Brayden, Trends Food Sci. Technol. 2016, 53, 90-101.

[14] N. Summerlin, E. Soo, S. Thakur, Z. Qu, S. Jambhrunkar, A. Popat, Int. J. Pharm. 2015, 479, $282-90$.

[15] Z. Ahmadi, R. Mohammadinejad, M. Ashrafizadeh, J. Drug Delivery Sci. Tecnol. 2019, 51, 591604.

[16] D. Pando, M. Matos, G. Gutiérrez, C. Pazos, Colloids Surf. B 2015, 128, 398-404.

[17] Gopi N. Devaraj, S. R. Parakh, Ravi Devraj, S. S. Apte, B. Ramesh Rao, D. Rambhau, J. Colloid Interface. Sci. 2002, 251, 360-65. 
[18] D. Pando, M. Beltrán, I. Girone, M. Matos, C. Pazos, Food Chem. 2015, 170, 281-87.

[19] J. S. Vankayala, S. N. Battula, R. Kandasamy, G. A. Mariya, M. E. E. Franklin, H. A. Pushpadass, L. Naik Naik, J. Mol. Liq. 2018, 261, 387-96.

[20] C. Caddeo, M. Manconi, M. C. Cardia, O. Díez-Sales, A. M. Fadda, C. Sinico, Int. J. Pharm. 2015, 484, 138-145.

[21] C. Caddeo, R. Pons, C. Carbone, X. Fernández-Busquets, C. Cardia, A. M. Maccioni, A. M. Fadda, M. Manconi, Carbohydr. Polym. 2017, 157, 1853-61.

[22] C. Caddeo, L. Pucci, M. Gabriele, C. Carbone, X. Fernández-Busquets, D. Valenti, R. Pons, A. Vassallo, A. M. Fadda, M. Manconi, Int.J. Pharm. 2018, 538, 40-47.

[23] D. J. McClements, J. Rao, Crit. Rev. Food Sci. Nutr. 2011, 51, 285-330.

[24] J. Zhou, M. Zhou, F. F. Yang, C. Y. Liu, R. L. Pan, Q. Chang, X. M. Liu, Y. H. Liao, Mol Pharm. 2015, 12, 1084-95.

[25] M. Nasr, Drug Deliv. 2016, 23, 1444-52.

[26] Q. Fang, C. Ma, Q. Xia, Integr. Ferroelectr. 2016, 169, 7-14.

[27] M. Herneisey, J. Williams, J. Mirtic, L. Liu, S. Potdar, C. Bagia, J. E. Cavanaugh, J. M. Janjic, Ther. Deliv. 2016, 7, 1-14.

[28] C. C. Yen, C. W. Chang, M. C. Hsu, Y. T. Wu, Int. J. Mol. Sci. 2017, 18, 1853-1867.

[29] G. Mamadou, C. Charrueau, J. Dairou, N. Limas Nzouzi, B. Eto, G. Ponchel, Int. J. Pharm. 2017, 512, 150-55.

[30] F. F. Yang, J. Zhou, X. Hu, Z. Q. Cong, C. Y. Liu, R. L. Pan, Q. Chang, X. M. Liu, Y. H. Liao, Eur. J. Pharm. Biopharm. 2018, 114, 303-9.

[31] G. Davidov-Pardo, D. J. Mc Clements, Food Chem. 2015, 167, 205-212.

[32] M. Matos, A. Laca, F. Rea, O. Iglesias, M. Rayner, G. Gutiérrez, J. Food Eng. 2018, 222, 20717.

[33] L. Dai, W. Zhu, R. Liu, C. Si, Part. Syst. Charact. 2018, 1700447, 1-9.

[34] D. J. McClements, Y. Li, Adv. Colloid Interface Sci. 2010, 159, 213-28.

[35] A. J. Choi, Y. Jo, Y. J. Cho, T. E. Kim, C. T. Kim, Food Eng. Prog. 2017, 212, 15-24.

[36] M. Matos, G. Gutiérrez, O. Iglesias, J. Coca, C. Pazos, J. Food Eng. 2015, 166, 212-20. 
[37] A. Acevedo-Fani, H. D. Silva, R. Soliva-Fortuny, O. Martín-Belloso, A. A. Vicente, Food Hydrocoll. 2017, 71, 207-15.

[38] M. Matos, G. Gutiérrez, L. Martínez-Rey, O. Iglesias, C. Pazos, J. Food Eng. 2018, 226, 73-81.

[39] S. Scalia, V. Trotta, V. Iannuccelli, A. Bianchi, Colloids Surf. B 2015, 135, 42-49.

[40] V. Trotta, B. Pavan, L. Ferraro, S. Beggiato, D. Traini, L. Gomes Des Reis, S. Scalia, A. Dalpiaz, Europ. J. Pharm. Biopharm. 2018, 127, 250-259.

[41] J. Wu, Y. Wang, H. Yang, X. Liu, Z. Lu, Carbohydr. Polym. 2017, 175, 170-77.

[42] J. Jin, F. Shi, Q. Li, P. Li, T. Chen, Y. Wang, Z. Wang, Proc. SPIE 9722, Colloidal nanoparticles for biomedical applications XI 2016, 9722, 97221D-1.

[43] P. Ramalingam, Y. T. Ko, Colloids Surf. B 2016, 139, 52-61.

[44] A. Singh, I. Ahmad, S. Ahmad, Z. Iqbal, F. J. Ahmad, Drug Dev. Ind. Pharm. 2016, 42, 1524 36.

[45] A. R. Neves, J. Fontes Queiroz, S. Reis, J. Nanobiotechnol. 2016, 14, 1-11.

[46] C. W. Chang, C. Y. Wong, Y. T. Wu, M. C. Hsu, Eur. J. Drug Metab. Pharmacokinet. 2017, 42, $239-49$.

[47] C. C. Koga, J. E. Andrade, M. G. Ferruzzi, Y. Lee, J. Food Sci. 2016, 81, 292-300.

[48] N. Pujara, S. Jambhrunkar, K. Y. Wong, M. McGuckin, A. Popat, J. Colloid Interface. Sci. 2017, $488,303-8$.

[49] F. Liu, D. Ma, X. Luo, Z. Zhang, L. He, Y. Gao, D. J. McClements, Food Hydrocoll. 2018, 79, $450-61$.

[50] C.Guo, J. Yin, D. Chen,_Carbohydrate Polymers 2018, 181, 1033-1037.

[51] L. Dai, R. Liu, L.-Q. Hu, Z.-F. Zou, C.-L. Si , ACS Sustainable Chem. Eng. 2017,5, 8241-8249.

[52] R. B. Friedrich, B. Kann, K. Coradini, H. L. Offerhaus, R. C. R. Beck, M. Windbergs, Eur. J. Pharm. Sci. 2015, 78, 204-13.

[53] N. Summerlin, Z. Qu, N. Pujara, Y. Sheng, S. Jambhrunkar, M. McGuckin, A. Popat, Colloids Surf. B 2016, 144, 1-7.

[54] E. Juère, J. Florek, M. Bouchoucha, S. Jambhrunkar, K. Yau Wong, A. Popat, F. Kleitz, Mol. Pharm. 2017, 14, 4431-41. 
[55] S. K. Singh, V. Makadia, S. Sharma, M. Rashid, S. Shahi, P. R. Mishra, M. Wahajuddin, J. R. Gayen, Drug Deliv. Transl. Res. 2017, 7, 395-407.

[56] C. Sinico, R. Pireddu, E. Pini, D. Valenti, C. Caddeo, A. M. Fadda, F. Lai, Planta Med., 2016, $83,476-481$.

[57] P. Negi, M. Aggarwal, G. Sharma, C. Rathore, G. Sharma, B. Singh, O. P. Katare, Biomed. Pharmacother. 2017, 88, 480-87.

[58] E. Soo, S. Thakur, Z. Qu, S. Jambhrunkar, H. S. Parekh, A. Popat, J. Colloid Interface Sci. 2016, $462,368-74$.

[59] R. Kumar, K. Kaur, S. Uppal, S. K. Mehta, Ultrason. Sonochem. 2017, 37, 478-89.

[60] J. Hao, J. Zhao, S. Zhang, T. Tong, Q. Zhuang, K. Jin, W. Chen, H. Tang, Colloids Surf. B 2016, $147,376-86$.

[61] S. W. El-Far, M. W. Helmy, S. N. Khattab, A. A. Bekhit, A. A. Hussein, A. O. Elzoghby, Nanomedicine 2018, 13, 1-20.

[62] V. P. Shah, G. L. Flynn, A. Yacobi, H. I. Maibach, C. Bon, N. M. Fleischer, T. J. Franz, S. A. Kaplan, J. Kawamoto, L. J. Lesko, J.-P. Marty, L. K. Pershing, H. Schaefer, J. A. Sequeira, S. P. Shrivastava, J. Wilkin, R. L. Williams., Pharm. Res. 1998, 15, 167-71.

[63] J. Lademann, U. Jacobi, C. Surber, H. J. Weigmann, J. W. Fluhr., Eur. J. Pharm. Biopharm. 2009, 72, 317-323.

[64] J. M. McClung, K. C. DeRuisseau, M. A. Whidden, H. Van Remmen, A. Richardson, W. Song, I. Vrabas, S. K. Powers., Exp. Physiol. 2010, 95, 222-31.

[65] J. Carvalho-Peixoto, R. Cardilo Alves, L. C. Cameron, Appl. Physiol. Nutr. Metab. 2007, 32, 1186-90.

[66] C. Marianecci, L. Di Marzio, F. Rinaldi, C. Celia, D. Paolino, F. Alhaique, S. Esposito, M. Carafa, Adv. Colloid Interface Sci. 2014, 205, 187-205.

[67] S. Moghassemi, A. Hadjizadeh, J. Control. Release 2014, 185, 22-36.

[68] Q. Fang, C. Ma, Q. Xia, Integr. Ferroelectr. 2016, 169, 7-14.

[69] M. Sivakumar, S. Y. Tang, K. W. Tan, Ultrasonic Sonochem. 2014, 21, 2069-83.

[70] M. K. Li, H. S. Fogler, J. Fluid Mech. 1978, 88, 499-511.

[71] M. K. Li, H. S. Fogler, J. Fluid Mech. 1978, 88, 513-28. 
[72] E. Piacentini, E. Drioli, L. Giorno, J. Membrane Sci. 2014, 468, 410-22.

[73] N. Recharla, M. Riaz, S. Ko, S. Park, J. Funct. Foods 2017, 39, 63-73.

[74] Z. H. Loh, A. K. Samanta, P. W. Sia Heng, Asian J. Pharm. 2015, 10, 255-74. 